Article

\title{
Evolution of Volatile Compounds and Spoilage Bacteria in Smoked Bacon during Refrigeration Using an E-Nose and GC-MS Combined with Partial Least Squares Regression
}

\author{
Xinfu $\mathrm{Li}^{1,2}{ }^{1}$, Jiancai Zhu ${ }^{1}$, Cong $\mathrm{Li}^{1,2}{ }^{1}$, Hua Ye ${ }^{1}$, Zhouping Wang ${ }^{1}$, Xiang $\mathrm{Wu}^{2}$ \\ and Baocai $\mathrm{Xu}$ 1,2,3,* \\ 1 School of Food Science and Technology, Jiangnan University, Wuxi 214122, China; lixinfu316@126.com (X.L.); \\ zjc01@163.com (J.Z.); cobralc@126.com (C.L.); yehua_2004@163.com (H.Y.); wangzp@jiangnan.edu.cn (Z.W.) \\ 2 State Key Laboratory of Meat Processing and Quality Control, Yurun Group, Nanjing 211806, China; \\ wuxiang985@126.com \\ 3 School of Food Science and Biology Engineering, Hefei University of Technology, Hefei 230009, China \\ * Correspondence: baocaixu@163.com; Tel.: +86-25-56677180
}

Received: 2 November 2018; Accepted: 8 December 2018; Published: 11 December 2018

\begin{abstract}
The changes in the concentration of volatile organic compounds (VOCs) and biogenic amines (BAs) in smoked bacon during 45-day refrigerated storage is investigated using solid-phase micro-extraction coupled with gas chromatography-mass spectrometry and high-performance liquid chromatography. In total, 56 VOCs and 6 BAs were identified and quantified. The possible pathways leading to their formation are analyzed and considered as the potential signs of microbial activity, especially by specific spoilage microorganisms (SSOs). Leuconostoc and Lactobacillus, which levels increased markedly with the extension of storage time, were recognized as SSOs. An electronic nose (e-nose) was employed to determine the changes in concentration of the odor components per sample present within half an hour. Partial least squares regression was then carried out to analyze the correlation between SSO growth, metabolite concentration, BA accumulation, and e-nose response. The results show that ten VOCs (ethanol, 2-furanmethanol, 1-hexanol, 1-propanol, phenol, 2-methoxyphenol, acetic acid, 3-ethyl-2-cyclopenten-1-one, furfural, and ethyl hexanoate) and three BAs (putrescine, cadaverine, and tyramine) can be associated with the growth of SSOs. Thus, they can be adopted as potential indicators to evaluate and monitor the quality of the bacon and develop appropriate detection methods. E-noses can used to recognize odors and diagnose quality of bacon.
\end{abstract}

Keywords: smoked bacon; volatile compounds; SPME-GC-MS; electronic nose; biogenic amines; partial least squares regression

\section{Introduction}

The spoilage of meat due to the growth of microorganisms and their associated metabolic processes, especially a group of bacteria called specific spoilage microorganisms (SSOs), is responsible for great economical losses in the food industry [1-5]. Numerous studies have reported that SSOs are associated with detrimental biochemical changes, e.g., production of volatile organic compounds (VOCs), accumulation of biogenic amines (BAs), etc. [1-3].

The growth and metabolism of SSOs in meat products during storage frequently leads to the production of off-flavors/odors, acidity, and gas [6-8]. Of course, certain volatile compounds in cooked meats contribute much to their acceptability [9]. However, the accumulation of VOCs (microbial metabolites) from SSOs, e.g., organic acids, alcohols, aldehydes, ketones, esters, 
sulfur compounds, and amines, determines the extent to which 'sensory spoilage' occurs and gradually causes a decline in quality characteristics of the meat [2,10-12]. At the same time, BAs can occur as a result of the accumulation of the products from SSOs, which also relate to food spoilage $[5,13,14]$.

The changes occurring in the microbiota and biochemistry of the meat during storage have been identified as a potential means of revealing the meat's quality or freshness [15-17]. For instance, butanoic acid and acetoin are related to the growth of lactic acid bacteria (LAB) and other spoilage microbial groups in packed beef [10]. Furthermore, the production of 3-methyl-1-butanol by SSOs (e.g., Pseudomonas spp. and Carnobacterium spp.) in meat stored under air and/or vacuum packaging is responsible for its off-odor [2]. In addition, Mansur et al. found that several microbial VOCs, such as acetic acid, butanoic acid, and 2-butanone, can be used as potential spoilage indicators [18]. The quantitative determination of BA profiles (mostly tyramine, putrescine, and cadaverine which are considered potentially toxic to humans) is an important method of ascertaining the quality and freshness of food [14,19]. Monitoring changes in the volatile fractions can also help in determining freshness and occurrence of microbial spoilage $[6,8,20]$.

Volatile fractions can be analyzed using solid-phase microextraction (SPME) coupled with gas chromatography and mass spectrometry (SPME-GC-MS). The SPME technique, for example, has been successfully applied to analyze the VOCs in different foodstuffs [21]. BAs in meat and meat products may be detected using high-performance liquid chromatography (HPLC). However, although SPME-GC-MS and HPLC are effective ways of identifying and quantifying chemical compounds such as flavors and liquid substances individually, they cannot distinguish among these samples in overall profiles [22].

Electronic noses (e-noses) are a relatively recent innovation which use an array of chemical sensors that can detect and recognize odors and therefore constitute an advantageous tool for the rapid and accurate diagnosis of food quality $[23,24]$. Used with appropriate mathematical methods, an e-nose has the ability to distinguish aroma patterns and monitor changes in odors/flavors and the results can be used for classification and quality control [25-27]. Studies have already demonstrated the potential use of e-noses to predict the shelf-life of packaged beef [23], milk [28], and raw materials [24]. Siegmund and Pfannhauser used an e-nose and GC-MS to investigate the changes of the volatile fraction of-cooked chicken during chill storage and obtained highly correlated results [29]. Arnold and Senter confirmed that an e-nose can be used to differentiate the VOCs produced by bacteria isolated from processed poultry, different bacterial species (e.g., Salmonella enteritidis, Escherichia coli) have different compounds as area percentage (e.g., alcohols and 1-propanol) [30]. However, very little research has focused on predicting the shelf-life of smoked bacon.

As a multivariate statistical method, partial least squares regression (PLSR) may be the optimal way of elucidating the correlations between multiple variables by reducing the dimensions of the original data set without losing any information [31]. A plane figure can be used to explain the differences and similarities between samples, and the corresponding load maps can be interpreted to discover what effects are responsible for the similarities between samples [32]. Therefore, PLSR analysis is more accurate and has great potential in quality control applications aimed at identifying spoilage.

Bacon is produced from belly pork and demand for it has increased markedly in China in recent years. However, its high moisture and nutrient content and mild heat treatment during production make it highly susceptible to microbial spoilage when it is stored and, as a result, the acceptability of the quality / flavour decreases progressively [10,21,33].

It is well known that spoilage in meat is related to detrimental biochemical changes. To the best of our knowledge, only a limited number of studies have been carried out to study the changes. In this article, the VOCs and BAs potentially produced by spoilage-associated microorganisms over a 45-day period of storage will be monitored and described and e-nose will be used to classify groups of samples, the correlation and contribution of them will be discussed. The main aim of this work was to identify several key substances related to deterioration for evaluating and monitoring the quality of bacon during storage and even for exploiting rapid, convenient, and sensitive detection methods. 


\section{Results and Discussion}

\subsection{Results of the E-Nose Analysis}

The e-nose is designed to detect and discriminate between complex odors according to the sensitivity ranges of the sensors. Properties such as shelf-life and freshness can thereby be investigated for any material that gives out volatile compounds [34,35].

In this article, the e-nose was used to monitor changes in the bacon's volatile compounds during storage. The mean sensor responses of the bacon are shown in Table S1. The radar chart shows how the average responses of the array of sensors differed with the samples (Figure 1a). The compounds that the sensors of the e-nose are sensitive to can therefore be investigated [36]. In general, the T- and P-type sensors gave responses that increased significantly $(p<0.05)$ with prolonged storage time, while the LY-sensors had responses that fell rapidly $(p<0.05)$. The response values may be used to infer which of the various volatile compounds are the principal ones resulting in the different responses of the e-nose and the different concentrations occurring during storage (the intensity of the corresponding sensor response increasing with increasing concentration).

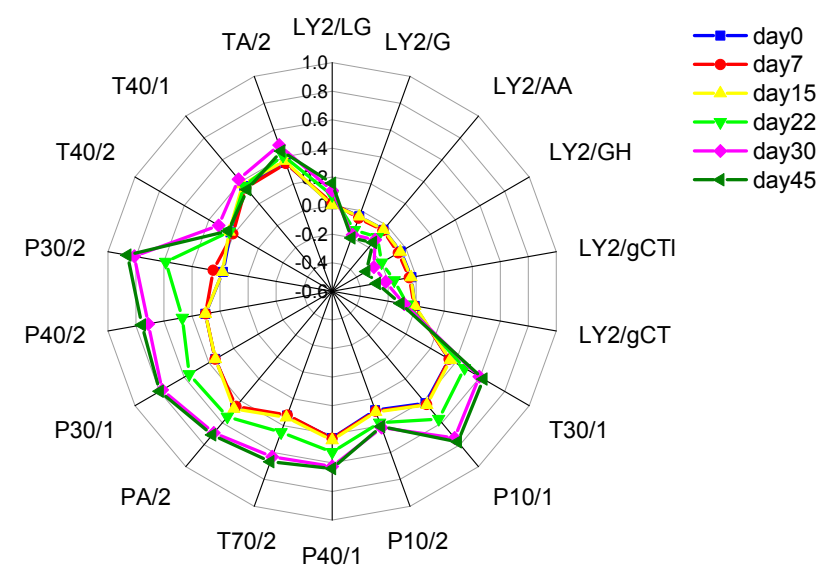

(a)

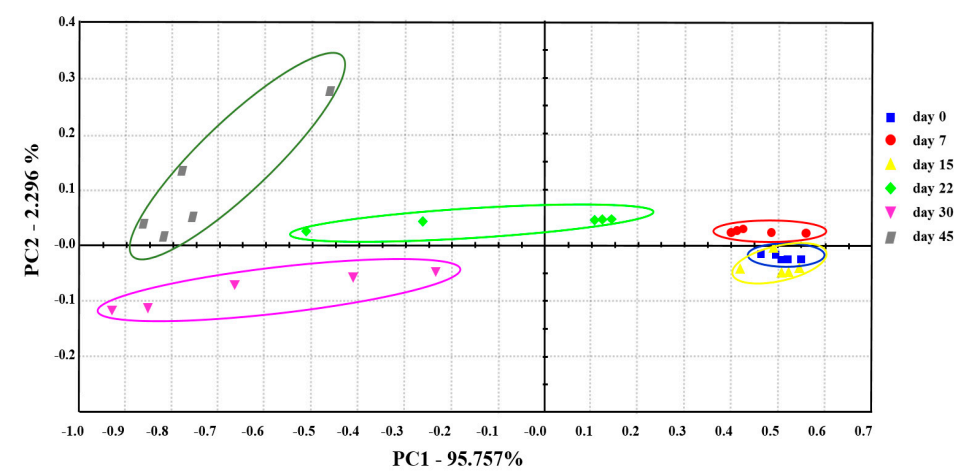

(b)

Figure 1. Results of radar plots and PCA of e-nose: (a) Radar plots of the mean responses of the sensors at different storage, each value represents the average score by five parallels with three replications. (b) Score plot using PCA analysis for discriminating bacon groups for different storage times at $4{ }^{\circ} \mathrm{C}$. All 18 metal sensors were used as the inputs of PCA, PC1 and PC2 accounted for 95.7\% and $2.3 \%$ of the variation, respectively. Each ellipse represents distances that are statistically equidistant from the group.

In order to understand the e-nose identification process and the repeatability of the smoked bacon samples, the PCA method was used to analyze the multivariate data based on variable quantity restriction (Figure 1b, Table S2). In the figure, the representative data points for the bacon are plotted 
in a space whose axes are based on the first and second major components (principal components PC1 and PC2, respectively). The first two components account for $98.05 \%$ of the accumulative variance, which covers almost all the variable information. The data points from the samples are distinctly clustered on days 0,7 , and 15 , indicating that the characteristic odors of these stored samples are very similar. However, the data points from day 22 are distributed within the first and second quadrants, a very different distribution to those of the previous samples. This indicates that the odor profile on day 22 was significantly different. Meanwhile, it is noteworthy that the points from day 30 are located in the third quadrant. Further change must have continued as the data points from day 45 were found to be distributed in the second quadrant. These results show that the e-nose performs well in identifying and differentiating between samples stored for over 15 days.

\subsection{Microbiological Growth and Specific Spoilage Microorganisms}

Microbiological data pertinent to the present analysis has been obtained in earlier research [37]. Briefly, the number of microorganisms increases sharply (by orders of magnitude) with prolonged storage. Culture-dependent experiments (traditional cultivation method) show that Leuconostoc mesenteroides and L. carnosum become the dominant species after 15 days. Culture-independent experiments (high-throughput sequencing (HTS)) indicate that LAB (Leuconostoc and Lactobacillus) prevail in the middle-late stages. These are considered to be SSOs. Carnobacterium, Vibrio Brochothrix, Serratia, Fusobacterium, Rahnella, and Lactococcus may also contribute to spoilage due to their proliferation or formation of metabolic products. The relative abundances of the top 10 species of spoilage bacteria at the genus level are shown in Table 1.

\subsection{GC-MS Results for Bacon after Different Storage Times}

For quantification purposes, a total of 56 calibration curves were drawn (Table 2). Among these compounds, phenols organic acids, alcohols and aldehydes, which had been reported as the most abundant compounds in Cantonese sausage [12], dry-cured ham [38], cold-smoked salmon [8], and fermented sausage [39-41]. According to Table 3, 56 volatile compounds were identified and quantified over the storage period, these compounds belong to eight classes: nine aldehydes, eight alcohols, 13 phenols, nine ketones, five alkanes, six terpenes, four organic acids, and two other compounds.

\subsection{Volatile Compound Evolution during Storage}

Although there were some small fluctuations, the amount of volatile compounds present tended to increase during storage (Table 3). The total amount fell to $644 \mathrm{mg} / \mathrm{kg}$ on day 7 having been $1100 \mathrm{mg} / \mathrm{kg}$ on day 0 ; it then increased to $3042 \mathrm{mg} / \mathrm{kg}$ on day 45. Table 3 shows that aldehydes and phenols were the major components in the initial stages of storage, followed by ketones. At the same time, alcohols, organic acids, alkanes, and others were minor components among the volatiles.

Considering the total occurrence of the compounds, the alcohol and phenol content increased dramatically during storage and clearly dominated the VOCs both in numbers and in amounts produced. At the same time, ketones, alkanes, organic acids, and terpenes exhibited minimal changes in their content. Only the aldehyde content appears to decrease as storage is prolonged.

Pearson correlation coefficients were used to reveal the correlations between the independent variables of storage time and VOC concentration. The results demonstrated that there were strong correlations between alcohols, phenols, and organic acids during refrigerated storage. More precisely, all 56 compounds were analyzed and a total of 23 were found to be significantly correlatedn, as can be seen from Table 3 . Of these 23 compounds, 21 showed positive correlation over the storage period, and the other two were negatively correlated. In particular, ethanol, 2-furan-methanol, 1-hexanol, and 1-propanol ( $r$ equal to 0.982, 0.982, 0.977, and 0.930, respectively) and phenol and 2-methoxyphenol ( $r$ equal to 0.956 and 0.919 , respectively) show a perfect positive correlation, as the bivariate correlation $(r)$ exceeds 0.900 [42]. The acetic acid $(r=0.964)$ and 3-ethyl-2-cyclopenten-1-one ( $r=0.916)$ levels in organic acids and ketones had a similar tendency. In contrast, of the 9 aldehydes present only furfural $(r=-0.900)$ was perfectly negatively correlated. 
Table 1. The relative abundances of the 10 top species of spoilage bacteria (at the genus level) in the bacon after $0,7,15,22,30$, and 45 days of refrigerated storage [37].

\begin{tabular}{|c|c|c|c|c|c|c|c|c|}
\hline \multirow{2}{*}{ Spoilage Bacteria } & \multicolumn{6}{|c|}{ Storage Time/Days } & \multicolumn{2}{|c|}{$\begin{array}{c}\text { Pearson's Correlation } \\
\text { Coefficients }\end{array}$} \\
\hline & Day 0 & Day 7 & Day 15 & Day 22 & Day 30 & Day 45 & $r$ & $p$ \\
\hline Leuconostoc & $0.91 \pm 0.44^{\mathrm{c}}$ & $1.74 \pm 0.44^{\mathrm{c}}$ & $26.72 \pm 15.09^{b}$ & $53.73 \pm 18.97^{a}$ & $40.26 \pm 21.17^{\mathrm{a}, \mathrm{b}}$ & $27.49 \pm 12.85^{b}$ & 0.616 & 0.193 \\
\hline Lactobacillus & $2.62 \pm 1.16^{\mathrm{c}}$ & $3.76 \pm 1.85^{\mathrm{c}}$ & $6.25 \pm 3.76^{c}$ & $9.45 \pm 8.48^{b, c}$ & $18.70 \pm 6.90^{b}$ & $41.94 \pm 16.14^{\mathrm{a}}$ & $0.934^{* *}$ & 0.006 \\
\hline Vibrio & $5.91 \pm 4.80^{\mathrm{b}}$ & $5.42 \pm 2.51^{b}$ & $30.69 \pm 30.27^{a}$ & $10.96 \pm 16.22^{a, b}$ & $8.08 \pm 14.84^{\mathrm{a}, \mathrm{b}}$ & $8.44 \pm 17.32^{\mathrm{a}, \mathrm{b}}$ & -0.022 & 0.968 \\
\hline Brochothrix & $2.43 \pm 0.73^{a, b}$ & $12.76 \pm 20.75^{a}$ & $1.48 \pm 0.48^{\mathrm{a}, \mathrm{b}}$ & $2.41 \pm 2.77^{\mathrm{a}, \mathrm{b}}$ & $0.17 \pm 0.07^{b}$ & $0.33 \pm 0.21^{b}$ & -0.527 & 0.282 \\
\hline Serratia & $0.34 \pm 0.33$ & $0.47 \pm 0.34$ & $0.20 \pm 0.07$ & $0.80 \pm 1.19$ & $6.28 \pm 12.89$ & $1.57 \pm 2.83$ & 0.48 & 0.335 \\
\hline Kocuria & $13.19 \pm 6.90^{\mathrm{a}}$ & $4.48 \pm 2.07^{b}$ & $1.56 \pm 1.15^{b}$ & $0.33 \pm 0.22^{b}$ & $0.38 \pm 0.14^{b}$ & $0.36 \pm 0.33^{b}$ & -0.76 & 0.079 \\
\hline Macrococcus & $8.58 \pm 9.59^{\mathrm{a}}$ & $0.97 \pm 0.29^{b}$ & $0.27 \pm 0.25^{b}$ & $0.01 \pm 0.00^{b}$ & $0.15 \pm 0.15^{b}$ & $0.01 \pm 0.00^{b}$ & -0.657 & 0.156 \\
\hline Carnobacterium & $1.00 \pm 0.37^{c}$ & $1.51 \pm 0.47^{\mathrm{c}}$ & $9.66 \pm 6.62^{a, b}$ & $12.94 \pm 8.14^{\mathrm{a}}$ & $12.15 \pm 6.38^{\mathrm{a}, \mathrm{b}}$ & $5.52 \pm 1.29^{b, c}$ & 0.48 & 0.335 \\
\hline Rahnella & $0.12 \pm 0.07$ & $0.48 \pm 0.55$ & $0.05 \pm 0.03$ & $0.13 \pm 0.13$ & $0.27 \pm 0.07$ & $2.78 \pm 5.73$ & 0.745 & 0.089 \\
\hline Fusobacterium & $1.02 \pm 1.18$ & $0.89 \pm 1.45$ & $0.16 \pm 0.20$ & $0.09 \pm 0.14$ & $3.58 \pm 0.36$ & $0.91 \pm 1.79$ & 0.261 & 0.618 \\
\hline Others & $63.88 \pm 2.93^{a}$ & $67.51 \pm 19.52^{a}$ & $22.98 \pm 14.39^{b}$ & $9.15 \pm 3.49^{b}$ & $9.97 \pm 2.61^{b}$ & $10.65 \pm 1.82^{b}$ & $-0.826^{*}$ & 0.043 \\
\hline
\end{tabular}

Figures in the table are means and standard error. ${ }^{a, b, c}$ Means within a row refer to the significant difference at $p<0.05$ according to Tukey's multiple range test. Means in the same row with no superscript letters after them or with a common superscript letter following them are not significantly different $(p<0.05)$. ${ }^{*}$ Significant at the 0.05 level; ${ }^{* *}$ Significant at the 0.01 level.

Table 2. The standard curve, validation range and coefficient of determination $\left(\mathrm{r}^{2}\right)$ for the volatile compounds in smoked bacon must. The equation based on the concentrations of peak areas and mean of six replicates at each of seven concentrations, total of 42 samples, " $y$ " represents the peak area ratio and " $x$ " indicates the concentration ratio. A model solution was used to test the quantities of the standards as described later.

\begin{tabular}{ccccc}
\hline No & Compound & Standard Curve & $\mathbf{r}^{\mathbf{2}}$ & Validation Range $\left(\boldsymbol{\mu \mathbf { ~ k g } ^ { - \mathbf { 1 } } )}\right.$ \\
\hline 1 & 3-Methylbutanal & $\mathrm{y}=1.11 \mathrm{x}+0.035$ & 0.987 & $0.1-5$ \\
2 & Hexanal & $\mathrm{y}=1.84 \mathrm{x}-0.019$ & 0.991 & $10-100$ \\
3 & Furfural & $\mathrm{y}=0.94 \mathrm{x}+0.083$ & 0.986 & $10-100$ \\
4 & Heptanal & $\mathrm{y}=1.1 \mathrm{x}+0.004$ & 0.992 & $1-50$ \\
5 & 5-Methyl-2-furancarboxaldehyde & $\mathrm{y}=0.88 \mathrm{x}+0.031$ & 0.982 & $1-50$ \\
6 & Octanal & $\mathrm{y}=0.73 \mathrm{x}-0.041$ & 0.984 & $1-100$ \\
7 & Phenylacetaldehyde & $\mathrm{y}=1.13 \mathrm{x}+0.051$ & 0.992 & $1-50$ \\
8 & Nonanal & $\mathrm{y}=2.12 \mathrm{x}+0.006$ & 0.979 & $20-500$ \\
9 & Decanal & $\mathrm{y}=0.95 \mathrm{x}-0.081$ & 0.981 & $1-50$ \\
10 & Ethanol & $\mathrm{y}=1.21 \mathrm{x}+0.065$ & 0.986 & $50-1000$ \\
11 & 1-Propanol & $\mathrm{y}=0.95 \mathrm{x}-0.027$ & 0.988 & $50-1000$ \\
12 & 2-Butanol & $\mathrm{y}=1.17 \mathrm{x}+0.094$ & 0.987 & $20-500$ \\
\hline
\end{tabular}


Table 2. Cont

\begin{tabular}{|c|c|c|c|c|}
\hline No & Compound & Standard Curve & $\mathrm{r}^{2}$ & Validation Range $\left(\mu \mathrm{g} \mathrm{kg}^{-1}\right)$ \\
\hline 13 & 3-Methyl-1-Butanol & $\mathrm{y}=1.18 \mathrm{x}+0.028$ & 0.992 & $1-10$ \\
\hline 14 & Propylene glycol & $y=1.24 x+0.007$ & 0.987 & $1-50$ \\
\hline 15 & 1-Pentanol & $y=0.81 x+0.057$ & 0.981 & $1-50$ \\
\hline 16 & 2-Furanmethanol & $y=1.15 x-0.025$ & 0.979 & $20-500$ \\
\hline 17 & 1-Hexanol & $y=2.28 x+0.068$ & 0.973 & $1-50$ \\
\hline 18 & Phenol & $y=1.51 x+0.029$ & 0.986 & $10-100$ \\
\hline 19 & 2-Methylphenol & $y=0.86 x+0.021$ & 0.992 & $10-100$ \\
\hline 20 & 4-Methylphenol ( $p$-cresol) & $y=0.98 x+0.009$ & 0.978 & $10-100$ \\
\hline 21 & 3-Methylphenol & $y=0.88 x+0.059$ & 0.979 & $1-50$ \\
\hline 22 & 2-Methoxyphenol & $\mathrm{y}=1.17 \mathrm{x}-0.061$ & 0.984 & $20-500$ \\
\hline 23 & 2,6-Dimethylphenol & $y=1.17 x-0.068$ & 0.991 & $1-10$ \\
\hline 24 & 2-Methoxy-3-methylphenol & $y=0.88 x+0.069$ & 0.992 & $1-10$ \\
\hline 25 & Creosol & $y=0.84 x+0.029$ & 0.994 & $20-500$ \\
\hline 26 & 4-Ethyl-2-methoxyphenol & $y=0.97 x-0.019$ & 0.984 & $10-100$ \\
\hline 27 & 2-Methoxy-4-vinylphenol & $y=2.13 x-0.029$ & 0.977 & $1-10$ \\
\hline 28 & 2,6-Dimethoxyphenol & $y=1.19 x-0.012$ & 0.975 & $10-100$ \\
\hline 29 & Eugenol & $y=1.16 x+0.068$ & 0.987 & $1-10$ \\
\hline 30 & trans-Isoeugenol & $y=0.96 x+0.043$ & 0.984 & $1-10$ \\
\hline 31 & 1-Hydroxy-2-propanone & $y=1.15 x+0.042$ & 0.986 & $1-50$ \\
\hline 32 & 2-Cyclopentenone & $y=0.93 x-0.051$ & 0.989 & $1-10$ \\
\hline 33 & 2-Methyl-2-cyclopentenone & $y=0.82 x-0.067$ & 0.978 & $10-100$ \\
\hline 34 & 3-Methyl-2-cyclopentenone & $y=1.19 x+0.018$ & 0.983 & $10-100$ \\
\hline 35 & 3,4-Dimethyl-2-cyclopentenone & $y=0.99 x+0.085$ & 0.984 & $0.1-5$ \\
\hline 36 & 2-Hydroxy-3-methyl-2-cyclopentenone & $y=0.96 x-0.027$ & 0.991 & $10-100$ \\
\hline 37 & 2,3-Dimethyl-2-cyclopentenone & $y=0.85 x+0.047$ & 0.978 & $10-100$ \\
\hline 38 & 3-Ethyl-2-cyclopentenone & $y=1.17 x+0.018$ & 0.979 & $1-50$ \\
\hline 39 & 3-Ethyl-2-hydroxy-2-cyclopentenone & $y=1.26 x-0.069$ & 0.992 & $1-50$ \\
\hline 40 & 2,3,4-Trimethylpentane & $y=1.14 x+0.068$ & 0.986 & $1-50$ \\
\hline 41 & 2,3,3-Trimethylpentane & $y=0.79 x-0.028$ & 0.985 & $1-50$ \\
\hline 42 & Octane & $y=0.86 x+0.021$ & 0.985 & $1-50$ \\
\hline 43 & Decane & $y=0.88 x+0.068$ & 0.977 & $1-50$ \\
\hline 44 & Cyclooctane & $y=1.18 x-0.012$ & 0.978 & $1-50$ \\
\hline 45 & 2,2,8-Trimethyldecane & $y=0.096 x+0.035$ & 0.986 & $1-50$ \\
\hline 46 & 3-Methyl-3-heptene & $\mathrm{y}=0.99 \mathrm{x}-0.039$ & 0.982 & $1-10$ \\
\hline 47 & (Z)-2-Octene & $y=0.86 x+0.017$ & 0.981 & $1-10$ \\
\hline 48 & Styrene & $y=0.94 x-0.008$ & 0.994 & $1-10$ \\
\hline 49 & $\alpha$-Pinene & $y=1.27 x+0.058$ & 0.989 & $0.1-5$ \\
\hline 50 & D-Limonene & $y=1.17 \cdot x-0.059$ & 0.995 & $1-10$ \\
\hline 51 & Acetic acid & $y=0.86 x+0.029$ & 0.981 & $20-500$ \\
\hline 52 & Butanoic acid & $\mathrm{y}=0.84 \mathrm{x}-0.046$ & 0.993 & $1-50$ \\
\hline 53 & Propanoic acid & $y=0.97 x+0.027$ & 0.976 & $0.1-5$ \\
\hline 54 & Benzoic acid & $y=0.86 x+0.038$ & 0.977 & $1-50$ \\
\hline 55 & 2-Furylmethylketone & $y=0.98 x-0.017$ & 0.988 & $1-10$ \\
\hline 56 & Ethyl hexanoate & $y=1.18 \cdot x+0.051$ & 0.992 & $1-10$ \\
\hline
\end{tabular}


Table 3. Concentrations of VOCs ( $\mathrm{mg} / \mathrm{kg}$ ) found for the bacon after $0,7,15,22,30$, and 45 days of refrigerated storage, as measured using GC-MS.

\begin{tabular}{|c|c|c|c|c|c|c|c|c|c|c|c|}
\hline \multirow[t]{2}{*}{ Code $^{A}$} & \multirow[t]{2}{*}{ Compound } & \multirow{2}{*}{$\begin{array}{c}\mathrm{RI}^{\mathrm{B}} \\
\text { (Calculated) }\end{array}$} & \multirow[t]{2}{*}{ Identification ${ }^{\mathrm{C}}$} & \multicolumn{6}{|c|}{ Storage Time/Days } & \multicolumn{2}{|c|}{$\begin{array}{c}\text { Pearson's } \\
\text { Correlation } \\
\text { Coefficients }\end{array}$} \\
\hline & & & & Day 0 & Day 7 & Day 15 & Day 22 & Day 30 & Day 45 & $r$ & $p$ \\
\hline & Aldehydes & & & & & & & & & & \\
\hline 1 & 3-Methylbutanal & 703 & MS, RI, Sta & $1.89 \pm 0.35^{\mathrm{a}, \mathrm{D}}$ & - & $1.58 \pm 0.55^{\mathrm{b}}$ & $1.82 \pm 0.35^{\mathrm{a}, \mathrm{b}}$ & $1.42 \pm 0.35^{\mathrm{c}}$ & - & -0.373 & 0.467 \\
\hline 2 & Hexanal & 803 & MS, RI, Sta & $97.61 \pm 10.77^{a}$ & $38.98 \pm 4.89^{\mathrm{c}}$ & $103.2 \pm 6.02^{\mathrm{a}}$ & $70.91 \pm 7.86^{b}$ & $47.01 \pm 10.08^{c}$ & $24.38 \pm 3.19^{d}$ & -0.644 & 0.167 \\
\hline 3 & Furfural & 839 & MS, RI, Sta & $84.36 \pm 7.13^{\text {a }}$ & $72.74 \pm 4.03 \mathrm{~b}$ & $72.36 \pm 8.49 \mathrm{~b}$ & $13.25 \pm 2.05^{c}$ & - & - & $\underset{* *}{-0.900}$ & 0.007 \\
\hline 4 & Heptanal & 904 & MS, RI, Sta & $33.7 \pm 5.60^{a}$ & $0.71 \pm 0.13^{c}$ & $8.22 \pm 1.52^{b}$ & - & $3.4 \pm 0.86^{c}$ & - & -0.639 & 0.172 \\
\hline 5 & 5-Methyl-2-Furan-carboxaldehyde & 969 & MS, RI, Sta & $14.72 \pm 2.26^{b}$ & $15.62 \pm 1.54 \mathrm{a}, \mathrm{b}$ & $17.45 \pm 0.90^{a}$ & $2.9 \pm 0.35^{c}$ & - & - & $\begin{array}{c}-0.844 \\
*\end{array}$ & 0.035 \\
\hline 6 & Octanal & 1006 & MS, RI, Sta & $88.70 \pm 7.82^{\text {a }}$ & $24.89 \pm 3.10^{\mathrm{e}}$ & $79.15 \pm 6.67^{\mathrm{b}}$ & $53.53 \pm 6.11^{\mathrm{c}}$ & $40.92 \pm 3.37^{\mathrm{d}}$ & $53.8 \pm 6.94^{\mathrm{c}}$ & -0.299 & 0.566 \\
\hline 7 & Phenyl-acetaldehyde & 1052 & MS, RI, Sta & $1.49 \pm 0.21 \mathrm{~b}$ & - & - & $2.88 \pm 0.60^{b}$ & $1.87 \pm 0.56^{b}$ & $36.98 \pm 6.27$ a & 0.779 & 0.068 \\
\hline 8 & Nonanal & 1107 & MS, RI, Sta & $234.35 \pm 28.98^{\mathrm{b}}$ & $59.21 \pm 7.11^{\mathrm{e}}$ & $384.74 \pm 27.84^{\mathrm{a}}$ & $161.42 \pm 20.22^{\mathrm{d}}$ & $151.19 \pm 16.09^{d}$ & $189.73 \pm 19.80^{c}$ & -0.069 & 0.897 \\
\hline \multirow[t]{3}{*}{9} & Decanal & 1209 & MS, RI, Sta & $13.72 \pm 2.67^{\mathrm{b}}$ & $3.94 \pm 0.38^{c}$ & $17.65 \pm 1.56^{a}$ & $2.54 \pm 0.33^{c}$ & $2.08 \pm 0.38^{c}$ & - & -0.65 & 0.162 \\
\hline & & & & $570.54 \pm 41.07^{b}$ & $216.09 \pm 6.69^{\mathrm{d}}$ & $684.35 \pm 40.11^{\mathrm{a}}$ & $309.25 \pm 29.37^{c}$ & $247.87 \pm 19.55$ & $304.89 \pm 23.41^{\mathrm{C}}$ & -0.421 & 0.406 \\
\hline & Alcohols & & & & & & & & & & \\
\hline 10 & Ethanol & $<700$ & MS, RI, Sta & $5.07 \pm 0.85^{d}$ & $24.61 \pm 2.72^{\mathrm{d}}$ & $123.68 \pm 12.65^{\mathrm{c}}$ & $265.77 \pm 39.00^{\mathrm{b}}$ & $414.96 \pm 52.38^{\text {a }}$ & $525.24 \pm 78.14^{\mathrm{a}}$ & $0.982 * *$ & 0 \\
\hline 11 & 1-Propanol & $<700$ & MS, RI, Sta & - & - & - & $75.73 \pm 10.402^{c}$ & $160.68 \pm 20.27^{b}$ & $381.37 \pm 48.94^{\mathrm{a}}$ & $0.930 * *$ & 0.007 \\
\hline 12 & 2-Butanol & $<700$ & MS, RI, Sta & - & - & $16.83 \pm 2.93^{b}$ & $13.54 \pm 3.66^{b}$ & - & $212.07 \pm 32.92^{\mathrm{a}}$ & 0.77 & 0.073 \\
\hline 13 & 3-Methyl-1-butanol & 735 & MS, RI, Sta & - & - & $6.06 \pm 0.86^{\mathrm{b}, \mathrm{c}}$ & $5.07 \pm 1.16^{c}$ & $10.44 \pm 1.24^{\mathrm{a}}$ & $7.56 \pm 1.69^{b}$ & $0.819 *$ & 0.046 \\
\hline 14 & Propylene Glycol & 753 & MS, Sta & $5.47 \pm 0.42^{\mathrm{d}}$ & $1.94 \pm 0.26^{\mathrm{e}}$ & $12.66 \pm 2.85^{\mathrm{b}}$ & $11.51 \pm 2.14^{\mathrm{b}, \mathrm{c}}$ & $6.81 \pm 1.02^{c, d}$ & $17.32 \pm 3.25^{a}$ & 0.74 & 0.093 \\
\hline 15 & 1-Pentanol & 767 & MS, RI, Sta & $0.59 \pm 0.15^{b}$ & - & - & $2.58 \pm 0.13 \mathrm{~b}$ & - & $37.43 \pm 3.14^{\mathrm{a}}$ & 0.764 & 0.077 \\
\hline 16 & 2-Furanmethanol & 861 & MS, RI, Sta & $27.39 \pm 5.05^{c}$ & $28.39 \pm 2.66^{c}$ & $63.5 \pm 3.70^{b}$ & $66.29 \pm 11.99^{b}$ & $66.83 \pm 9.28^{b}$ & $113.76 \pm 13.50^{\mathrm{a}}$ & $0.982 * *$ & 0.003 \\
\hline \multirow[t]{3}{*}{17} & 1-Hexanol & 871 & MS, RI, Sta & $1.37 \pm 0.18^{c}$ & $1.14 \pm 0.12^{c}$ & $15.41 \pm 1.29^{b}$ & $38.12 \pm 2.62^{a}$ & $37.23 \pm 7.35^{a}$ & $41.24 \pm 6.73^{\mathrm{a}}$ & $0.905 * *$ & 0.013 \\
\hline & & & & $39.89 \pm 4.73^{\mathrm{d}}$ & $56.08 \pm 1.62^{d}$ & $238.15 \pm 16.41^{\mathrm{d}}$ & $478.61 \pm 41.06^{\mathrm{c}}$ & $696.95 \pm 61.40^{\mathrm{b}}$ & $1335.99 \pm 106.72^{a}$ & $0.976^{* *}$ & 0.001 \\
\hline & Phenols & & & & & & & & & & \\
\hline 18 & Phenol & 990 & MS, RI, Sta & $10.33 \pm 2.98^{c}$ & $12.4 \pm 2.01^{\mathrm{c}}$ & $18.62 \pm 2.97^{c}$ & $28.51 \pm 4.10^{b}$ & $49.11 \pm 9.57^{\mathrm{a}}$ & $52.62 \pm 6.54^{\mathrm{a}}$ & $0.956^{* *}$ & 0.003 \\
\hline 19 & 2-Methylphenol & 1064 & MS, RI, Sta & $16.41 \pm 2.04^{b}$ & $19.1 \pm 1.48^{\mathrm{b}}$ & $37.76 \pm 4.63^{\mathrm{a}}$ & $41.73 \pm 6.39$ a & $33.58 \pm 5.52^{a}$ & $41.74 \pm 8.47^{a}$ & 0.796 & 0.058 \\
\hline 20 & 4-Methylphenol (p-cresol) & 1086 & MS, RI, Sta & $41.52 \pm 4.48^{c}$ & $34.74 \pm 3.78^{c}$ & $70.97 \pm 7.45^{-a, b}$ & $45.12 \pm 5.20^{c}$ & $54.16 \pm 12.28^{b}$ & $76.45 \pm 7.96^{a}$ & 0.706 & 0.117 \\
\hline 21 & 3-Methylphenol & 1088 & MS, RI, Sta & $2.11 \pm 0.37^{\mathrm{d}}$ & - & $12.1 \pm 1.42^{\mathrm{c}}$ & $45.13 \pm 4.26^{\mathrm{a}}$ & $22.99 \pm 3.49^{b}$ & $26.09 \pm 4.39 \mathrm{~b}$ & 0.644 & 0.168 \\
\hline 22 & 2-Methoxyphenol & 1092 & MS, RI, Sta & $134.97 \pm 16.79^{\mathrm{d}}$ & $118.61 \pm 11.19^{d}$ & $280.97 \pm 26.87^{\mathrm{c}}$ & $293.44 \pm 21.90^{\mathrm{b}, \mathrm{c}}$ & $331.51 \pm 44.66^{\mathrm{a}, \mathrm{b}}$ & $372.9 \pm 47.34^{\mathrm{a}}$ & $0.919^{* *}$ & 0.01 \\
\hline 23 & 2,6-Dimethyl-phenol & 1114 & MS, RI, Sta & - & - & - & - & - & $7.23 \pm 0.83$ & 0.758 & 0.081 \\
\hline 24 & 2-Methoxy-3-methylphenol & 1191 & MS, Sta & $1.16 \pm 0.25^{\mathrm{d}}$ & $1.34 \pm 0.16^{\mathrm{d}}$ & $4.98 \pm 0.75^{b}$ & $3.49 \pm 0.32 \mathrm{~b}, \mathrm{c}$ & $3.1 \pm 0.83^{c}$ & $8.19 \pm 0.99 \mathrm{a}$ & 0.850 * & 0.032 \\
\hline
\end{tabular}


Table 3. Cont

\begin{tabular}{|c|c|c|c|c|c|c|c|c|c|c|c|}
\hline \multirow[t]{2}{*}{ Code $^{\mathrm{A}}$} & \multirow[t]{2}{*}{ Compound } & \multirow[t]{2}{*}{$\begin{array}{c}\mathrm{RI}^{\mathrm{B}} \\
\text { (Calculated) }\end{array}$} & \multirow[t]{2}{*}{ Identification ${ }^{C}$} & \multicolumn{6}{|c|}{ Storage Time/Days } & \multicolumn{2}{|c|}{$\begin{array}{l}\text { Pearson's } \\
\text { Correlation } \\
\text { Coefficients }\end{array}$} \\
\hline & & & & Day 0 & Day 7 & Day 15 & Day 22 & Day 30 & Day 45 & $r$ & $p$ \\
\hline 25 & Creosol & 1194 & MS, RI, Sta & $49.2 \pm 6.94^{d}$ & $34.45 \pm 3.68^{d}$ & $95.11 \pm 18.12^{b, c}$ & $108.1 \pm 9.56^{\mathrm{b}}$ & $69.6 \pm 9.31^{c}$ & $123.38 \pm 18.34^{\mathrm{a}}$ & 0.768 & 0.074 \\
\hline 26 & 4-Ethyl-2-methoxyphenol & 1282 & MS, RI, Sta & $18.29 \pm 2.57^{\mathrm{d}}$ & $13.72 \pm 1.98^{\mathrm{d}}$ & $35.96 \pm 3.68^{b, c}$ & $41.85 \pm 6.86^{a, b}$ & $29.86 \pm 3.16^{\mathrm{c}}$ & $42.23 \pm 9.39^{\mathrm{a}}$ & 0.759 & 0.08 \\
\hline 27 & 2-Methoxy-4-vinylphenol & 1319 & MS, RI, Sta & $5.16 \pm 1.09^{\mathrm{c}}$ & $2.17 \pm 0.44^{\mathrm{d}}$ & $7.92 \pm 1.24^{\mathrm{a}}$ & $8.03 \pm 1.51 \mathrm{a}, \mathrm{b}$ & $5.47 \pm 0.77 \mathrm{~b}, \mathrm{c}$ & $8.66 \pm 1.46^{\mathrm{a}}$ & 0.613 & 0.196 \\
\hline 28 & 2,6-Dimethoxy-phenol & 1356 & MS, Sta & $26.45 \pm 2.63^{\mathrm{b}}$ & $16.67 \pm 1.81^{\mathrm{b}}$ & $52.52 \pm 8.82^{a}$ & $54.18 \pm 8.64^{\mathrm{a}}$ & $42.58 \pm 6.82^{\mathrm{a}}$ & $53.58 \pm 9.49^{a}$ & 0.699 & 0.122 \\
\hline 29 & Eugenol & 1362 & MS, RI, Sta & $1.25 \pm 0.15^{\mathrm{d}}$ & $0.29 \pm 0.06^{\mathrm{d}}$ & $2.55 \pm 0.30^{c}$ & $12.66 \pm 1.53^{\mathrm{a}}$ & - & $4.4 \pm 0.63^{\mathrm{b}}$ & 0.251 & 0.631 \\
\hline \multirow[t]{3}{*}{30} & trans-Isoeugenol & 1458 & MS, RI, Sta & $1.27 \pm 0.15^{c}$ & - & $3.48 \pm 0.73^{a}$ & $3.05 \pm 0.47 \mathrm{a}, \mathrm{b}$ & $2.98 \pm 0.89 \mathrm{a}, \mathrm{b}$ & $2.51 \pm 0.37^{b}$ & 0.539 & 0.27 \\
\hline & & & & $308.12 \pm 20.85^{\mathrm{c}}$ & $253.48 \pm 8.10^{\mathrm{c}}$ & $622.94 \pm 37.94^{\mathrm{b}}$ & $685.31 \pm 22.89^{\mathrm{b}}$ & $644.94 \pm 79.80^{\mathrm{b}}$ & $819.97 \pm 87.33^{\mathrm{a}}$ & $0.894^{*}$ & 0.016 \\
\hline & Ketones & & & & & & & & & & \\
\hline 31 & 1-Hydroxy-2-propanone & 669 & MS, RI, Sta & $17.23 \pm 2.23^{b}$ & $12.6 \pm 2.64^{b, c}$ & $41.36 \pm 6.70^{\mathrm{a}}$ & $9.21 \pm 0.94^{c}$ & - & - & -0.548 & 0.26 \\
\hline 32 & 2-Cyclopentenone & 839 & MS, RI, Sta & - & - & - & $8.46 \pm 1.41$ & $7.9 \pm 0.96$ & $8.55 \pm 1.84$ & $0.847^{*}$ & 0.033 \\
\hline 33 & 2-Methyl-2-cyclo-pentenone & 910 & MS, RI, Sta & $17.3 \pm 2.41^{\mathrm{c}}$ & $15.59 \pm 1.62^{\mathrm{c}}$ & $42.62 \pm 6.15^{\mathrm{b}}$ & $43.09 \pm 6.86^{\mathrm{b}}$ & $38.02 \pm 4.25^{\mathrm{b}}$ & $58.92 \pm 5.99^{a}$ & $0.895^{*}$ & 0.016 \\
\hline 34 & 3-Methyl-2-cyclo-pentenone & 971 & MS, RI, Sta & $19.03 \pm 2.31^{c}$ & $20.6 \pm 2.30^{b, c}$ & $34.75 \pm 4.76^{a}$ & $25.02 \pm 4.11^{\mathrm{b}}$ & $36.99 \pm 8.94^{a}$ & $40.73 \pm 3.54^{\mathrm{a}}$ & $0.867^{*}$ & 0.025 \\
\hline 35 & 3,4-Dimethyl-2-cyclopentenone & 1027 & MS, Sta & - & - & - & $3.47 \pm 0.75$ & - & - & 0.065 & 0.902 \\
\hline 36 & 2-Hydroxy-3-methyl-2-cyclo-pentenone & 1034 & MS, RI, Sta & $15.23 \pm 2.22^{\mathrm{c}}$ & $3.66 \pm 0.51^{\mathrm{d}}$ & $40.12 \pm 5.12^{b}$ & - & $36.85 \pm 9.58^{\mathrm{b}}$ & $61.47 \pm 7.86^{\mathrm{a}}$ & 0.708 & 0.115 \\
\hline 37 & 2,3-Dimethyl-2-cyclopentenone & 1042 & MS, RI, Sta & $17.43 \pm 1.69^{\mathrm{c}}$ & $16.12 \pm 1.65^{\mathrm{c}}$ & $40.2 \pm 6.53^{\mathrm{b}}$ & $43.6 \pm 7.56^{\mathrm{b}}$ & $33.79 \pm 4.66^{\mathrm{b}}$ & $52.96 \pm 6.07^{\text {a }}$ & $0.855^{*}$ & 0.03 \\
\hline 38 & 3-Ethyl-2-cyclopentenone & 1080 & MS, Sta & $3 \pm 0.32^{\mathrm{c}}$ & $3.43 \pm 0.30^{c}$ & $6.53 \pm 1.47^{\mathrm{b}, \mathrm{c}}$ & $6.12 \pm 0.98^{b, c}$ & $7.52 \pm 1.32^{b}$ & $17.91 \pm 2.63^{\mathrm{a}}$ & $0.916 *$ & 0.01 \\
\hline \multirow[t]{2}{*}{39} & 3-Ethyl-2-hydroxy-2-cyclo-pentenone & 1100 & MS, RI, Sta & $12.25 \pm 1.59^{c}$ & $8.03 \pm 2.62^{\mathrm{d}}$ & $17.45 \pm 2.04^{b}$ & $11.3 \pm 1.64^{\mathrm{c}}$ & $12.49 \pm 1.83^{\mathrm{c}}$ & $26.83 \pm 4.06^{\mathrm{a}}$ & 0.732 & 0.098 \\
\hline & & & & $101.47 \pm 3.70^{\mathrm{e}}$ & $80.01 \pm 7.17^{\mathrm{f}}$ & $223.03 \pm 22.43^{\mathrm{b}}$ & $146.8 \pm 11.78^{\mathrm{d}}$ & $173.56 \pm 25.92^{\mathrm{c}}$ & $267.38 \pm 15.01^{\mathrm{a}}$ & 0.807 & 0.052 \\
\hline 40 & $\begin{array}{c}\text { Alkanes } \\
\text { 2,3,4-Trimethyl-Pentane }\end{array}$ & 752 & MS, Sta & - & - & $3.11 \pm 0.62^{\mathrm{c}}$ & $6.81 \pm 1.77^{\mathrm{b}}$ & $1.42 \pm 0.30^{\mathrm{c}}$ & $15.85 \pm 2.77^{\mathrm{a}}$ & $0.833^{*}$ & 0.04 \\
\hline 41 & 2,3,3-Trimethyl-pentane & 759 & MS, Sta & - & - & - & - & $3.37 \pm 0.69$ & $33.35 \pm 4.28$ & 0.801 & 0.055 \\
\hline 42 & Octane & 801 & MS, RI, Sta & $2.54 \pm 0.33^{\mathrm{c}}$ & - & $17.39 \pm 2.94^{\mathrm{b}}$ & $37.18 \pm 5.44^{\mathrm{a}}$ & $16.16 \pm 2.54^{\mathrm{b}}$ & $20.49 \pm 3.47^{\mathrm{b}}$ & 0.572 & 0.236 \\
\hline 43 & Decane & 1000 & MS, RI, Sta & $19.72 \pm 1.99^{c}$ & $3.48 \pm 0.48^{\mathrm{d}}$ & $13.4 \pm 2.24^{c}$ & $72.29 \pm 7.58^{a}$ & $48.09 \pm 7.18^{b}$ & $22.49 \pm 3.45^{c}$ & 0.343 & 0.506 \\
\hline 44 & Cyclooctane & 1075 & MS, Sta & $1.24 \pm 0.23^{c}$ & $3.12 \pm 0.41^{b}$ & $4.41 \pm 1.46^{b}$ & - & - & $15.02 \pm 1.82^{\mathrm{a}}$ & 0.631 & 0.179 \\
\hline
\end{tabular}


Table 3. Cont.

\begin{tabular}{|c|c|c|c|c|c|c|c|c|c|c|c|}
\hline \multirow[t]{2}{*}{ Code $\mathrm{A}^{\mathrm{A}}$} & \multirow[t]{2}{*}{ Compound } & \multirow{2}{*}{$\begin{array}{c}\mathrm{RI}^{\mathrm{B}} \\
\text { (Calculated) }\end{array}$} & \multirow[t]{2}{*}{ Identification $\mathrm{C}$} & \multicolumn{6}{|c|}{ Storage Time/Days } & \multicolumn{2}{|c|}{$\begin{array}{c}\text { Pearson's } \\
\text { Correlation } \\
\text { Coefficients }\end{array}$} \\
\hline & & & & Day 0 & Day 7 & Day 15 & Day 22 & Day 30 & Day 45 & $r$ & $p$ \\
\hline & & & & $23.5 \pm 1.94 \mathrm{e}^{\mathrm{e}}$ & $6.6 \pm 0.74^{\mathrm{f}}$ & $38.31 \pm 1.32^{d}$ & $116.28 \pm 11.83^{a}$ & $69.03 \pm 6.12^{c}$ & $107.2 \pm 5.38^{\mathrm{b}}$ & 0.701 & 0.12 \\
\hline \multicolumn{12}{|c|}{ Terpene compounds } \\
\hline 45 & 2,2,8-Trimethyl-Decane & 792 & MS, Sta & - & - & $5.04 \pm 1.23^{b}$ & $0.99 \pm 0.53^{c}$ & $4.28 \pm 0.49^{b}$ & $18.03 \pm 3.03^{\mathrm{a}}$ & $0.850 *$ & 0.032 \\
\hline 46 & 3-Methyl-3-heptene & 798 & MS, Sta & - & - & - & - & $3.62 \pm 0.75$ & $8.13 \pm 1.17$ & $0.855^{*}$ & 0.019 \\
\hline 47 & (Z)-2-Octene & 807 & MS, RI, Sta & - & - & - & - & - & $5.15 \pm 0.79$ & 0.758 & 0.081 \\
\hline 48 & Styrene & 898 & MS, RI, Sta & - & - & - & $10.44 \pm 1.65$ & $3.57 \pm 0.34$ & - & 0.172 & 0.745 \\
\hline 49 & $\alpha$-Pinene & 940 & MS, RI, Sta & - & - & $0.91 \pm 0.23^{b}$ & $0.92 \pm 0.14^{b}$ & $1.95 \pm 0.38^{a}$ & $0.55 \pm 0.12^{c}$ & 0.523 & 0.287 \\
\hline \multirow[t]{3}{*}{50} & D-Limonene & 1036 & MS, RI, Sta & - & $0.88 \pm 0.17^{c}$ & - & - & $5.74 \pm 0.83^{b}$ & $8 \pm 1.42^{a}$ & $0.867^{*}$ & 0.025 \\
\hline & & & & - & $0.88 \pm 0.17^{\mathrm{e}}$ & $5.95 \pm 1.09^{d}$ & $15.82 \pm 2.09^{c}$ & $19.16 \pm 0.60^{\mathrm{b}}$ & $39.87 \pm 3.97^{\mathrm{a}}$ & $0.975^{* *}$ & 0.001 \\
\hline & Organic acids & & & & & & & & & & \\
\hline 51 & Acetic acid & $<700$ & MS, RI, Sta & $24.78 \pm 3.14^{\mathrm{e}}$ & $10.72 \pm 2.71^{\mathrm{f}}$ & $44.88 \pm 6.66^{\mathrm{d}}$ & $64.73 \pm 9.02^{c}$ & $100.71 \pm 11.31^{\mathrm{b}}$ & $126.69 \pm 16.48^{a}$ & $0.964^{* *}$ & 0.002 \\
\hline 52 & Butanoic acid & 890 & MS, RI, Sta & $9.89 \pm 0.85^{\mathrm{c}}$ & $2.62 \pm 0.50^{\mathrm{d}}$ & $9.36 \pm 1.87^{c}$ & $3.68 \pm 0.92^{d}$ & $22.57 \pm 2.97^{\mathrm{a}}$ & $16.22 \pm 1.10^{b}$ & 0.6 & 0.208 \\
\hline 53 & Propanoic acid & 985 & MS, RI, Sta & - & - & - & - & $0.64 \pm 0.17$ & $1.10 \pm 0.19$ & $0.890 *$ & 0.017 \\
\hline \multirow[t]{3}{*}{54} & Benzoic acid & 1549 & MS, RI, Sta & $12.26 \pm 2.56^{\mathrm{b}, \mathrm{c}}$ & $1.79 \pm 0.20^{\mathrm{e}}$ & $15.22 \pm 3.47^{\text {a.b }}$ & $16.71 \pm 4.48^{a}$ & $9.73 \pm 1.98^{c, d}$ & $8.26 \pm 1.74^{\mathrm{d}}$ & 0.008 & 0.989 \\
\hline & & & & $46.93 \pm 4.70^{\mathrm{f}}$ & $19.39 \pm 2.87^{\mathrm{e}}$ & $69.46 \pm 7.13^{\mathrm{d}}$ & $85.12 \pm 7.60^{c}$ & $133.65 \pm 13.43^{b}$ & $152.27 \pm 14.41^{\mathrm{a}}$ & $0.935^{* *}$ & 0.006 \\
\hline & Others & & & & & & & & & & \\
\hline 55 & 2-Furylmethyl-ketone & 914 & MS, RI, Sta & - & $11.26 \pm 1.21$ & $9.11 \pm 1.15$ & - & - & - & -0.437 & 0.386 \\
\hline \multirow[t]{3}{*}{56} & Ethyl hexanoate & 998 & MS, RI, Sta & - & - & - & $2.97 \pm 0.90^{c}$ & $5.05 \pm 1.09^{b}$ & $14.28 \pm 1.67^{\mathrm{a}}$ & $0.920 * *$ & 0.009 \\
\hline & & & & - & $11.26 \pm 1.21^{\mathrm{b}}$ & $9.11 \pm 1.15^{c}$ & $2.97 \pm 0.90^{\mathrm{e}}$ & $5.05 \pm 1.09^{d}$ & $14.28 \pm 1.67^{a}$ & 0.523 & 0.287 \\
\hline & & & & $1090.45 \pm 47.93^{\mathrm{d}}$ & $643.79 \pm 13.25^{\mathrm{e}}$ & $1891.29 \pm 127.58 b$ & $1840.16 \pm 51.26^{\mathrm{c}}$ & $1990.21 \pm 207.91^{b}$ & $3041.85 \pm 140.65^{\mathrm{a}}$ & $0.918 * *$ & 0.01 \\
\hline
\end{tabular}

A Code representing the 56 volatile compounds used in the PISR analysis. ${ }^{B}$ The retention index of volatile compounds on DB- 5 columns. ${ }^{C}$ Method of identification: MS, mass spectrum comparison using Wiley library; RI, retention index in agreement with literature value; Sta, confirmed by authentic standards. ${ }^{\mathrm{D}}$ Figures in the table are means and standard error. The letters ${ }^{\mathrm{a}-\mathrm{f}}$ refer to the significant difference at $p<0.05$ according to Tukey's multiple range test. Means in the same row with no superscript letters after them or with a common superscript letter following them are not significantly different $(p<0.05)$. - Not detected in sample. The letter " $r$ " represents the relationship value between the variables of different storage days and the concentrations of VOCs, -1 to 0 is negative correlation, 0 no correlation, and 0 to +1 positive correlation. The letter " $p$ " refer to the value of the significant difference. * Significant at the 0.05 level; ** Significant at the 0.01 level. 


\subsection{Compounds Formed Related to Microbial Activity}

To gain a better understanding of how flavor is generated in the bacon, it is necessary to identify the sources of the volatile compounds. The production of VOCs in meat products is mainly a result of thermally-induced reactions and microbial activity. The former, for instance, include fatty acid oxidation and the Maillard reaction [43], both of which depend on the processing temperature and time (as well as other factors, such as, the addition of spices, smoking, the raw materials, and brining) $[40,41,44,45]$. Generally, the corruption of meat is mostly caused by the microbial populations of Enterobacteriaceae, LAB, Pseudomonads, Brochothrix thermosphacta, and some Clostridia [2].

Aldehydes are believed to make an important contribution to the flavor of smoked bacon, due to their high concentrations and low thresholds [46,47]. Linear aldehydes can be produced by the oxidation of lipid volatiles during processing (and degradation during storage) so that a wide range of flavors and odors can be formed [6,12]. Microorganisms associated with corruption, e.g., Pseudomonas spp., Carnobacterium spp., and Enterobacteriaceae are able to produce the highest number of aldehydes in the storage process [2].

In the samples used in this article, nine aldehydes (mainly the linear aldehydes including nonanal, hexanal, octanal, and furfural) were present in large amounts in the early stages and constituted the largest proportion of the VOCs measured. Straight-chain aldehydes (e.g., nonanal, hexanal, and heptanal) can be produced by Carnobacterium spp. and Enterobacteriaceae bacteria which are found in HTS. However, these compounds, with the exception of the phenylacetaldehyde, significantly decrease in abundance during storage $(p<0.05)$. The behavior of aldehydes association with spoilage bacteria is difficult to understand because they continued to fall in abundance-it may, however, be simply because these aldehydes were rapidly oxidized to acids and subsequently esterified with ethanol [12,48].

Alcohols are potentially produced by SSOs such as LAB (Leuconostoc, Lactobacillus), Carnobacterium spp., Pseudomonas spp., Staphylococcus, and Kocuria during the storage of meat $[2,4,8]$. Metabolic pathways, such as amino acid metabolism, lipid oxidation, methyl ketone reduction, and proteolytic activity are related to the alcohols generated $[2,12,49]$. As the quality of the bacon degraded with increasing storage time, nearly all of the nine alcohols increased significantly $(p<0.05)$ in abundance (e.g., see results for 1-hexanol in Table 3). The alcohols 3-methyl-1-butanol and 1-hexanol have been found inoculated with Enterococcus spp., Carnobacterium spp., C. divergens, and R. aquatilis. However, their low concentrations indicate that they did not play crucial roles in the spoilage process. The most abundant alcohols are ethanol, 2-furanmethanol, 1-propanol, and 2-butanol, which may be produced by LAB.

Phenols and methoxyphenols are compounds frequently encountered in wood smoke [45], and are formed by the pyrolysis of lignin [41]. In the smoked bacon studied in this article, eight phenols and five methoxyphenols were found (one predominant component is 2-methoxyphenol, which comes from softwood biomasses) which agrees with the results of other studies [41,50]. During vacuum storage, these compounds (especially 2-methoxyphenol) increased significantly in number $(p<0.05)$ and were positively correlated. This increase may also be related to growth of bacterial cells, such as LAB (especially enterococci and lactococci) which play a positive role in the production of phenol from benzaldehyde and phenylalanine $[6,41]$.

Ketones are often considered to be secondary products formed during lipid oxidation, alkane degradation, and dehydrogenation of secondary alcohols by bacteria [2,8]. The existence of the ketones is mostly connected with the presence of Staphylococcus, Pseudomonas spp., Enterobacteriaceae, and Carnobacterium spp. In our experiments, seven derivatives of 2-cyclopenten-1-one and 2 methyl ketones were found, most likely originating from the Maillard reaction from complex carbohydrates involved during pyrolysis [51], or from incomplete $\beta$-oxidation [52]. In this paper, the low ketone content and their slightly increasing amounts may indicate that they could be correlated with the Carnobacterium spp., Serratia, and Rahnella revealed by HTS.

The volatile fatty acids in the meat may have arisen from the hydrolysis of triglycerides and phospholipids [53]. The main source of volatile fatty acids in meat storage is Br. Thermosphacta, 
Carnobacterium spp., and LAB [2]. In this work, only five organic acids were detected during storage. The main ones are acetic and butanoic acids, which showed significant increases in abundance $(p<0.05)$ and were positively correlated with storage time. Acetic and butyric acids can be derived from the glucose catabolism of hetero-fermentative LAB, e.g., Leuconostoc carnosum, Lactobacillus spp., and the microbial transformation of threonine, which is commonly associated with meat spoilage [6,54-56]. In this study, LAB (principally Leuconostoc and Lactobacillus) increased dramatically in the bacon during storage [37]. Similar results have been obtained for sliced cooked pork shoulder [44] and cold-smoked salmon [57] during refrigerated storage.

The alkanes present can be ignored as they contribute little to the flavor [58]. The terpenoids are expected to have originated from the addition of brine [41,44]. However, they are not expected to be major contributors to the flavor due to their low concentrations.

\subsection{BA Evolution during Storage}

Changes in histamine, putrescine, cadaverine, tyramine, 2-phenylethylamine, tryptamine, spermidine, and spermine in smoked bacon during storage were determined following the HPLC method. The BA content of the smoked bacon during the storage period is shown in Table 4 . Neither tryptamine or phenylethylamine were found in any of the samples and remained absent throughout the entire storage period, a result that has been found before in Spanish meat products and chicken meat [59].

Table 4. BA concentrations $(\mathrm{mg} / \mathrm{kg})$ in the bacon after at $0,7,15,22,30$, and 45 days of refrigerated storage.

\begin{tabular}{|c|c|c|c|c|c|c|c|c|}
\hline \multirow[t]{2}{*}{$\begin{array}{l}\text { Biogenic } \\
\text { Amines }\end{array}$} & \multicolumn{6}{|c|}{ Storage Time/Days } & \multicolumn{2}{|c|}{$\begin{array}{l}\text { Pearson's } \\
\text { Correlation } \\
\text { Coefficients }\end{array}$} \\
\hline & Day 0 & Day 7 & Day 15 & Day 22 & Day 30 & Day 45 & $r$ & $p$ \\
\hline Phenylethylamine & - & - & - & - & - & - & - & - \\
\hline Putrescine & $2.81 \pm 0.57^{c, d}$ & $2.39 \pm 0.36^{\mathrm{d}}$ & $3.83 \pm 0.58^{b, c}$ & $2.81 \pm 0.70^{c, d}$ & $4.14 \pm 0.47^{\mathrm{b}}$ & $11.69 \pm 1.86^{\mathrm{a}}$ & $0.827 *$ & 0.042 \\
\hline Cadaverine & $5.75 \pm 0.53^{c}$ & $4.23 \pm 0.46^{\mathrm{d}}$ & $5.34 \pm 0.60^{c}$ & $7.50 \pm 0.93^{b}$ & $8.76 \pm 1.11^{\mathrm{a}}$ & $9.50 \pm 0.94^{\mathrm{a}}$ & $0.892 *$ & 0.017 \\
\hline Tyramine & $4.02 \pm 0.79^{c}$ & $4.51 \pm 0.93^{\mathrm{c}}$ & $4.21 \pm 0.53^{c}$ & $9.94 \pm 1.15^{b}$ & $14.06 \pm 1.93^{\mathrm{a}}$ & $15.94 \pm 2.48^{\mathrm{a}}$ & $0.940 *$ & 0.005 \\
\hline Spermidine & $2.22 \pm 0.45^{\mathrm{a}, \mathrm{b}}$ & $2.45 \pm 0.42^{\mathrm{a}}$ & $2.20 \pm 0.32^{\mathrm{a}, \mathrm{b}}$ & $2.22 \pm 0.30^{\mathrm{a}, \mathrm{b}}$ & $2.29 \pm 0.31^{a, b}$ & $1.85 \pm 0.15^{b}$ & -0.716 & 0.110 \\
\hline Spermine & $6.26 \pm 0.98$ & $7.40 \pm 0.77$ & $6.23 \pm 0.58$ & $7.02 \pm 1.05$ & $7.16 \pm 1.12$ & $6.93 \pm 0.69$ & 0.336 & 0.515 \\
\hline Total & $22.44 \pm 1.22^{\mathrm{d}}$ & $22.10 \pm 3.90^{\mathrm{d}}$ & $23.70 \pm 1.47^{\mathrm{d}}$ & $30.65 \pm 2.05^{c}$ & $38.58 \pm 3.93^{b}$ & $48.29 \pm 3.83^{\mathrm{a}}$ & $0.963 * *$ & 0.002 \\
\hline
\end{tabular}

Figures in the table are means and standard error. ${ }^{a-d}$ Means within a row refer to the significant difference at $p<0.05$ according to Tukey's multiple range test. Means in the same row with no superscript letters after them or with a common superscript letter following them are not significantly different $(p<0.05)$. - Not detected in sample.

* Significant at the 0.05 level; ${ }^{* *}$ Significant at the 0.01 level.

Spermidine and spermine, which were detected at concentrations that remained relatively stable, are amines that occur naturally in meat products [59,60]. Pearson correlation was used to determine the relationship between the independent variables of storage time and BA concentration. In general, the BAs (except spermidine) were found to be positively correlated with storage time, especially putrescine, cadaverine, and tyramine which increased significantly over the 45 days $(p<0.05)$. According to the literature, the accumulation of BAs in bacon appears to originate as a result of bacterial activity [59]. For example, Enterobacteriaceae (containing Serratia liquefaciens and Hafnia alvei), Staphylococci, Enterococci, Pseudomonas spp., and certain Lactobacilli, are reported to play a key role in the generation of putrescine, cadaverine, and tyramine in meat products $[14,19,61]$. In addition, Pediococcus strains are often responsible for the presence of histamine [62]. Furthermore, LAB and Enterobacteriaceae have been associated with the production of tyramine and cadaverine, respectively, in fermented sausages [63]. Dietary polyamine and putrescine accumulation is mainly due to the activity of microorganisms, including pseudomonads and Enterobacteriaceae [60].

In the work reported here, LAB dominated the middle-late stages and caused the production of decarboxylases and this led to an increasing tyramine concentration during storage. 
Enterobacteriaceae increased significantly on days 30 and 45 which may be responsible for the large amounts of putrescine and cadaverine detected on these days. The histamine content remained stable which may be due to the absence of Pediococcus and Pseudomonas aeruginosa.

\subsection{Relationships between Samples, VOCs, E-Nose Sensors, and Spoilage Bacteria}

We now consider the possible correlations between the characteristics of the gas sensors, e-nose identification results, variations in VOCs and BAs, and the change in spoilage bacteria present. To do this, the mean values accumulated from the physicochemical indicators and microbial characteristics were processed using ANOVA-PLSR.

The PLSR analysis involved using a total of 90 compounds as variables. The $X$-matrix was designated as the 18 e-nose sensors, 56 volatile compounds, six BAs, and 10 spoilage bacteria (Figure 2). The $Y$-matrix was generated using the variables scores after different storage periods. The output PLSR model consists of two vital PCs, which illustrate $81 \%$ of the variance of the cross-validation. Most of the variables are located between a small and a large ellipse $\left(\mathrm{r}^{2}=0.5\right.$ and 1.0 , respectively), which shows that the data is well described by the PLSR model [64].

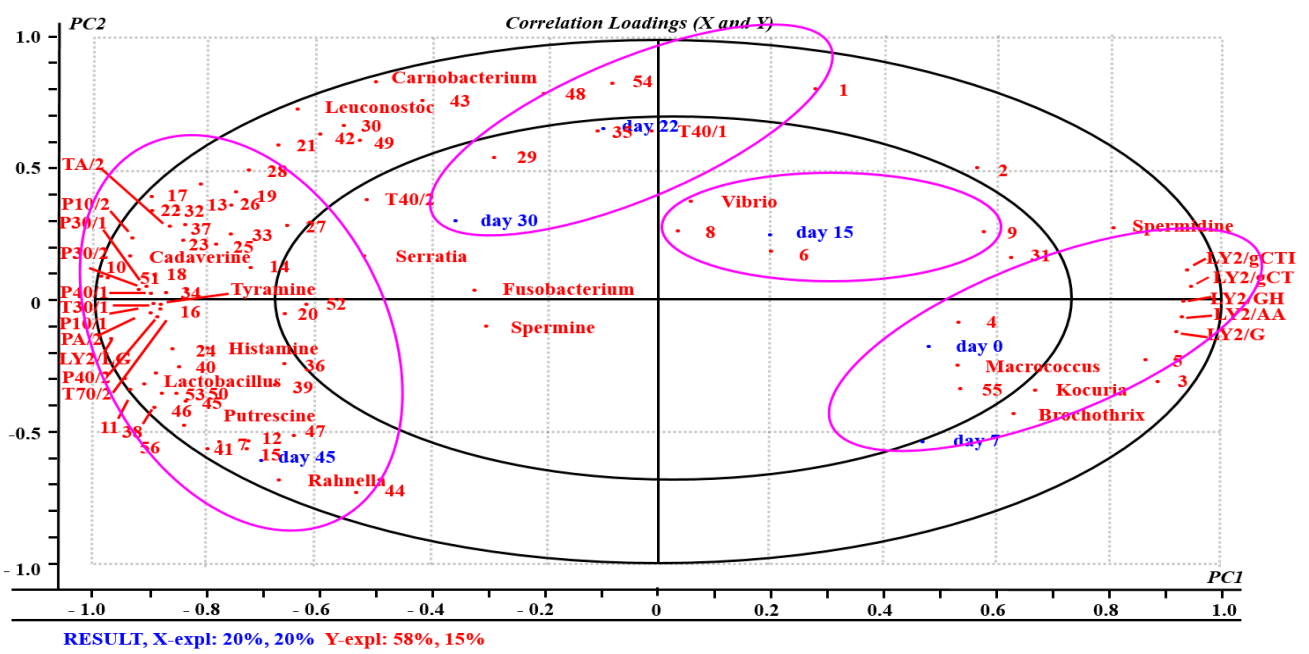

Figure 2. An overview of the variation found in the mean data from the partial least squares regression (PLSR) correlation loadings plot for bacon samples. The model was derived from e-nose sensors, VOCs, BAs and spoilage bacteria as the $X$-matrix and score values at different storage time as the $Y$-matrix. The concentric ellipses represent 100 and 50\% explained variance, respectively. The code in PLSR corresponds to the 56 volatile compounds in Table 3. The 18 e-nose sensors are given in Figure 1a and Table S1. The regions within the purple ellipses are highly correlated with different variables.

As indicated in Figure 2, the samples can be divided into four groups, considering their locations, the resultant correlation between PC1 and PC2, and storage time. Firstly, the samples on days 0 and 7 lie in the positive region of PC1 and negative region of PC2. It is easy to see that the LY2 gas sensors (except LY2/LG), spermidine, Brochothrix, Kocuria, and Macrococcus are distributed in the same region surrounded mainly by aldehydes (furfural and 5-methyl-2-furancarboxaldehyde). Thus, these variables have good correspondence in the initial storage period before day 7 . This phenomenon may be explained as follows. Most spoilage microorganisms are eliminated and inhibited by the mild heat treatment used during processing. The number of microbial counts did not differ significantly in the initial storage period showing that they could not be contributing to the formation of flavor substances. That is, the flavors and liquids present were mainly those naturally present in the meat product. Therefore, these results indicate that the original smoked bacon was of excellent quality.

Secondly, day 15 is located in the positive region of PC1 and PC2. It only seems to co-vary with aldehydes and Vibrio, and this is due to the situation being similar to that before day 7 . These results are in accordance with the results of the PCA analysis of the e-nose data (see Figure 1b). 
Thirdly, day 22 and day 30 lie in the negative region of PC1 and positive region PC2 surrounded by volatile compounds that are mainly alkanes, terpenes, and phenols, and correlated significantly with spoilage bacteria such as Serratia and Fusobacterium. The only BA nearby is spermine, and the data is well described by just two sensors: T40/1 and T40/2.

Fourthly, the samples on day 45 are in the negative region of PC1 and PC2. They co-varied with the responses of the T-type (excluding T40/1 and T40/2), P-type, and LY2-LG gas sensors, and are negatively correlated with the LY2 gas sensors (except LY2/G). These results indicate that the sensors have a great impact on differentiation between sample groups.

The results show that the e-nose can distinguish between and identify bacon in different stages of storage using the 18 sensors. Previous studies have shown that T- and P-type sensors are markedly related to sensory attributes such as off-flavors and odors $[65,66]$. Off-flavors/odors can be considered as being produced by SSO metabolites, are associated with lots of VOCs and usually contain aldehydes, alcohols, ketones, and others [2,67]. According to Figure 2, day 45 is significantly correlated with most of the volatile compounds (mainly alcohols, phenols, ketones and organic acids). As discussed previously in the article, they are spoilage-associated compounds produced by SSOs [2]. Combining this with the Pearson correlation analysis, the key VOCs are mainly 2-furan-methanol, ethanol, phenol, 2-methoxyphenol, and acetic acid. These compounds have higher concentrations and are significantly correlated $(p<0.05)$ with storage time. This shows that they make a greater contribution to flavor of the bacon as storage was prolonged.

Figure 2 also shows that day 45 co-varies with Lactobacillus, Rahnella, Carnobacterium, and Leuconostoc. These can thus be identified as the SSOs related to the spoilage of the bacon during storage. The current results are in good accord with those of Casaburi et al. [2] who proposed that Enterobacteriaceae and LAB are the SSOs associated with the spoilage of meat and production of many VOCs. Like other researchers, Rahnella [68], Carnobacterium spp. [69], and Leuconostoc spp. [70] have been found to play dominant roles (as SSOs) in the production of volatile compounds causing spoilage.

According to Figure 2, along the PC1 axis, BA accumulation mainly involves putrescine, histamine, tyramine, and cadaverine, which are located to the left of the plot and are positively correlated with the samples on day 45 and SSOs. BAs can be used as indicators of meat quality because they are metabolites derived from microbial growth and metabolism [59]. Therefore, the freshness of the bacon can be reflected by creating chemical quality and BA indices based on the measured levels of the BAs present (histamine, cadaverine, putrescine, and tyramine). As a result, early spoilage of the meat can be detected.

\section{Materials and Methods}

\subsection{Sampling and Storage Conditions}

Commercial samples of vacuum-packed smoked bacon were provided by Yurun (a joint venture between China and Italy whose factory is located in Ma'anshan in China). Bacon was produced from liquid smoke and sampled immediately after packaging. Samples were sampled along the processing line and vacuum packed individually, and transferred to the lab with drikold (dry ice). Then, the packages were thawed and refrigerated (at $0-4{ }^{\circ} \mathrm{C}$ ). Five different batches of manufactured bacon were sampled and for each production batch eighteen parallel samples were selected randomly, a total of ninety samples of smoked bacon were tested in experiments. The vacuum-packaged bacon produced on that day was immediately analyzed (corresponding to 'day zero'); the others after 7, 15, 22, 30, and 45 days of refrigerated storage (at $0-4{ }^{\circ} \mathrm{C}$ ). On each sampling points, samples were withdrawn in triplicate for the subsequent biochemical analyses. The specifications of the manufacturer are as follows: sizes of polyethylene bag $110 \times 220 \mathrm{~mm}$, slice thickness $2.5 \mathrm{~mm}, 8-9$ slices and $200 \mathrm{~g}$ per bag. 


\subsection{Electronic Nose}

The e-nose employed (a Fox 4000, Alpha MOS, Toulouse, France) was furnished with an HS100 autosampler (Alpha MOS), 18 metal oxide sensors, and propriety data processing software $\mathrm{n}$ and was used to analyze the volatile compounds from the bacon. The 18 sensors specifically used are referred to as LY2/AA, LY2/G, LY2/gCT, LY2/gCTL, LY2/GH, LY2/LG, P10/1, P10/2, P30/1, P30/2, P40/1, $\mathrm{P} 40 / 2, \mathrm{PA} / 2, \mathrm{~T} 30 / 1, \mathrm{~T} 40 / 2, \mathrm{~T} 70 / 2, \mathrm{~T} 40 / 1$, and TA/2.

Minced samples $(3.0 \mathrm{~g})$ were placed in a $10 \mathrm{~mL}$ glass vial. The headspace generation were incubated at $50{ }^{\circ} \mathrm{C}$ for $10 \mathrm{~min}$. Headspace gas $(2000 \mu \mathrm{L})$ was pumped into the sensor chamber for $10 \mathrm{~s}$ at a constant rate of $150 \mathrm{~mL} \mathrm{~min}^{-1}$. The injection volume was $800 \mu \mathrm{L}$ and the injected speed syringe was $2500 \mathrm{~mL} \mathrm{~min}^{-1}$. The sensor-response data were acquired for $120 \mathrm{~s}$ and the time between injections was set to $600 \mathrm{~s}$.

On each sampling point $(0,7,15,22,30$, and 45 days), samples were withdrawn in triplicate for the sub-sequent analyses, each sample was analyzed in three times. A total of ninety samples were tested in experiments. Based the data of the triplicate and three replications, the average results were used for PCA analysis to obtain a stable result. The software was used to calculate the discrimination index-the higher the index, the better the difference [71].

\subsection{Chemicals}

Authentic standards were obtained from commercial sources: 3-methylbutanal, hexanal, furfural, heptanal, octanal, phenylacetaldehyde, $\alpha$-pinene, 5-methyl-2-furancarboxaldehyde, nonanal, decanal, ethanol, 1-propanol, 2-butanol, 3-methyl-1-butanol, propylene glycol, 1-pentanol, 2-furanmethanol, 1-hexanol, phenol, 2-methylphenol, 4-methylphenol ( $p$-cresol), 3-methylphenol, 2-methoxyphenol, 2,6-dimethylphenol, 2-methoxy-3-methylphenol, creosol, 4-ethyl-2-methoxyphenol, 2-methoxy-4-vinylphenol, 2,6-dimethoxyphenol, octane, eugenol, trans-isoeugenol, 1-hydroxy-2-propanone, 2-cyclopentenone, cyclooctane, 2-methyl-2-cyclopentenone, 3,4-dimethyl-2-cyclo-pentenone, benzoic acid, 2-hydroxy-3-methyl-2-cyclopentenone, 3-methyl-2-cyclopentenone, acetic acid, 2,3-dimethyl-2-cyclopentenone, 3-ethyl-2-cyclopentenone, butanoic acid, styrene, 3-ethyl-2-hydroxy-2-cyclopentenone, 2,3,4-trimethylpentane, 2-furylmethylketone 2,3,3-trimethylpentane, 2,2,8-trimethyldecane, propanoic acid, 3-methyl-3-heptene, (Z)-2-octene, D-limonene, and ethyl hexanoate were purchased from Sigma-Aldrich (Shanghai, China). 2-Octanol (internal standard) and $n$-alkane standards $\left(\mathrm{C}_{6}-\mathrm{C}_{30}\right)$ were purchased from Sigma-Aldrich Chemical Co. (St. Louis, MO, USA). All substances are AR, at least $97 \%$ purity. The purification system from Milli-Q (Millipore, Bedford, MA, USA) to obtain purified water.

\subsection{SPME-GC-MS of Volatile Organic Compounds in Bacon}

A $4.0 \mathrm{~g}$ sample of minced bacon was put into a $20 \mathrm{~mL}$ glass vial. The bottle was sealed with a Teflon cover and placed in a water bath at $40^{\circ} \mathrm{C}$ for $15 \mathrm{~min}$. Extraction was performed using a previously-described method, with some slight modification [45]. A $65 \mu \mathrm{m}$ polydimethylsiloxane/divinylbenzene SPME-fiber was exposed to the headspace of the sample (with stirring) at $40{ }^{\circ} \mathrm{C}$ for $40 \mathrm{~min}$. It was then inserted into the thermal desorption system at $250{ }^{\circ} \mathrm{C}$ for $5 \mathrm{~min}$.

GC-MS analysis was performed using a GC system (Agilent 7890) equipped with SPME and a mass-selective detector (MSD; type 5975, Agilent Technologies, Palo Alto, CA, USA). Samples were analyzed on DB- 5 column $(60 \mathrm{~m} \times 0.25 \mathrm{~mm} \times 0.25 \mu \mathrm{m}$; Agilent). The injection port was programmed to $250{ }^{\circ} \mathrm{C}$, then retained for $5 \mathrm{~min}$, and then the sample was injected in splitless mode. The carrier gas (helium; purity $=99.999 \%$ ) flowed at a rate of $1.0 \mathrm{~mL} \mathrm{~min}^{-1}$. The MSD was used for chemical identification. The ionization energy voltage was set to $70 \mathrm{eV}$ and the temperatures of the ion source, quadrupole mass filter, and transfer line to 230,150 , and $250{ }^{\circ} \mathrm{C}$, respectively. The total ion current was monitored to record the chromatograms, and the scanning range was $40-450 \mathrm{~m} / \mathrm{z}$. The volatile compounds were identified according to the retention indices, retention times with those obtained for authentic standards, or with appropriate mass spectra libraries (Wiley, MD, USA, NIST, 2011). 
The $\mathrm{C}_{6}-\mathrm{C}_{30}$ saturated alkanes standard mixture was used for calculating retention indices (RIs). Each sample was subjected to GC-MS analysis in triplicate.

\subsection{Calibration of Standard Curves}

To get a matrix similar to bacon, model solution was prepared containing $30 \mathrm{mg} / \mathrm{g}$ hexadecanoic acid, $15 \mathrm{mg} / \mathrm{g}$ stearic acid, $30 \mathrm{mg} / \mathrm{g}$ oleic acid, $15 \mathrm{mg} / \mathrm{g}$ linoleic acid, $40 \mathrm{mg} / \mathrm{g}$ Glu, $40 \mathrm{mg} / \mathrm{g}$ Ala and $40 \mathrm{mg} / \mathrm{g}$ His in Milli-Q deionized water [52,72].

Seven levels of calibration: 1:5, 1:10, 1:20, 1:30, 1:40, 1:50 and 1:100 strengths were generated in triplicate. 2-Octanol ( $400 \mathrm{mg} \mathrm{L}^{-1}$ in water, $\left.500 \mu \mathrm{L}\right)$ was introduced into $4 \mathrm{~g}$ of model solution in a $20-\mathrm{mL}$ vial and then extracted by SPME, using the same conditions as for bacon.

The calibration curves for determination of volatile compounds in extracts by GC-MS after SPME was established. Each point is the mean of six replicates. The standard curves were shown in the research (Table 2), where y represented the peak area ratio (peak area of volatile standard/peak area of internal standard, $\mathrm{A}_{\mathrm{x}} / \mathrm{A}_{\mathrm{i}}$ ) and $\mathrm{x}$ represented the concentration ratio (concentration of volatile standard/concentration of internal standard, $C_{x} / C_{i}$ ). Fifty six volatile compounds were quantified to construct the standard curves [73].

\subsection{Biogenic Amine Determination}

Standard amine solutions were prepared according to previously developed procedures [74]. All the standard chemicals were obtained from Sigma Chemical Co. (St Louis, MO, USA) and CNW (Darmstadt, Germany). The extraction, purification, and separation steps were carried out according to the methods previously reported [14].

A Waters Alliance e2695 HPLC instrument fitted with a Waters UV-visible 2489 detector (Waters Co., Milford, MA, USA), and a Merck-Hitachi D-2500 chromato-integrator. The column used for separation was a Lichrospher 100RP-C18 (4.6 mm i.d. $\times 150 \mathrm{~mm}, 5 \mu \mathrm{m}$, Merck, Darmstadt, Germany). The BAs were detected using radiation of wavelength $254 \mathrm{~nm}$. The concentrations of eight BAs (histamine, putrescine, cadaverine, tyramine, 2-phenylethylamine, tryptamine, spermidine, and spermine) were thus determined. All analyses were carried out in triplicate and the quantitative unit used was $\mathrm{mg}$ amine $/ \mathrm{kg}$.

\subsection{Microbiological Analyses}

The method of culture-dependent to the present analysis can been obtained in earlier research [37]. Briefly, eight culture media at different temperatures and atmosphere conditions were used to cultivate different microbial groups and/or species during the storage of bacon. Then colonies were isolated from selective culture media and identified by the bacterial $16 \mathrm{~S} r$ RNA sequence analysis. Gene fragment was amplified with the universal primers 27F/1492R.

The method of culture-independent can also been obtained in the previous research [37]. Concisely, DNA extraction directly from bacon samples, pyrosequencing for 16S V3-V4 rRNA were conducted by using primer pairs $341 \mathrm{~F}$ and $802 \mathrm{R}$. Illumina sequencing was performed at Novogene Bioinformatics Technology Co., Ltd. (Beijing, China). Then the pyrosequence data was analysis.

\subsection{Quality Assurance/Quality Control (QA/QC)}

Both QA/QC are essential for the proper functioning of an analytical laboratory and the integrity of the data it produces. No interference was detected in the blanks, parallel and duplicates of the routine analytical procedures for bacon samples. The instrument was calibrated daily with calibration standard. The relative deviation between the concentration point of the calibration curve and the actual value was $<20 \%$, otherwise the calibration curve will be redrawn. The recoveries of the target analytes obtained from real samples based on internal standard were in the range of $80-115 \%$, and the relative standard deviation (RSD) was $<7 \%$. All concentrations were normalized and not corrected by surrogate recoveries. Adding low concentration mixed standard reserve solution to blank sample. The limits of 
detection (LODs, $\mathrm{S} / \mathrm{N}=3$ ) were $0.2-12.2 \mu \mathrm{g} / \mathrm{kg}$, and the limits of quantification (LOQs, $\mathrm{S} / \mathrm{N}=10$ ) were $2.5-35.3 \mu \mathrm{g} / \mathrm{kg}$. The QA/QC of HPLC is performed in the same way as GC/MS. The average percent recoveries ranging from $70 \%$ to $110 \%$. The LODs and the LOQs values were set as $0.05-0.5 \mathrm{mg} / \mathrm{kg}$ and $0.08-1.7 \mathrm{mg} / \mathrm{kg}$, respectively.

\subsection{Statistical Analysis}

The data from the experiments was analyzed using analysis of variance (ANOVA) tests and Pearson's correlation coefficient ' $r$ ' was employed to test the relationships among the different variables. Calculations were performed using the statistical package SPSS v20.0 (SPSS Inc., Chicago, IL, USA). Post-hoc multiple comparisons were determined by the Tukey's test with the level of significance set at $p<0.05$. PLSR models were adopted to study the correlation of the physicochemical and microbial characteristics and their contribution to the storage periods. The 18 sensors, 56 volatile compounds, 6 BAs, and 10 spoilage bacteria as the X-matrix and the variables scores at different storage periods as the $Y$-variable. PLSR analyses were performed using Unscrambler software (v9.7, CAMO, ASA, Oslo, Norway)—details of the procedures used are as described previously [65].

\section{Conclusions}

The shelf-life of meat and meat products is frequently assessed by performing sensory analyses and monitoring changes in microbial levels over time. However, there are some disadvantages to such an approach: analyses can be misleading, lengthy, expensive, and destructive. The measurement generated by the electronic nose can be associated to classification, determination of bacon evolution. It can potentially be commonly used by institutions, manufacturers and even consumers tasked with food quality control. It is also sensible to consider monitoring changes in some of the chemical metabolites produced during meat spoilage as a potential tool for assessing quality. A rapid analysis method/tool is needed to accurately quantify the chosen indicators used to predict the remaining shelf-life of food products. Therefore, we traced the evolution of VOCs, BAs, and associated microbial populations in smoked bacon during a period of refrigerated storage. A subsequent PLSR analysis showed which SSOs were closely related to the biochemical changes associated with spoilage. As a result, it was found that key roles are played by ethanol, 2-furanmethanol, 1-hexanol, 1-propanol, phenol, 2-methoxyphenol, acetic acid, 3-ethyl-2-cyclopenten-1-one, furfural, and ethyl hexanoate as well as putrescine, cadaverine, and tyramine. These key compounds can therefore be potentially used as the indicators for evaluating the quality of bacon and predicting its remaining storage time. They can also be used for developing rapid, convenient, and sensitive detection methods, such as gas-phase and liquid-phase biosensors for on-pack shelf-life determination. Therefore, before these biosensors are developed and applied in industry, it is necessary to study and analyze the metabolites and bacteria more deeply.

Supplementary Materials: The Supporting Information is available: Table S1: The mean sensor responses of bacon at $0,7,15,22,30$ and 45 days. Table S2: Loadings of 18 variables (e-nose sensors) on two significant principal components for smoked bacon.

Author Contributions: Conceptualization, B.X. and X.L.; Methodology, Z.W.; Software, C.L.; Validation, X.W.; Formal Analysis, X.L.; Investigation, X.W.; Resources, B.X.; Data Curation, X.W.; Writing-Original Draft Preparation, X.L. and H.Y.; Writing-Review \& Editing, X.L. and C.L.; Visualization, J.Z.; Supervision, J.Z.; Project Administration, X.W.; Funding Acquisition, B.X. All authors approved the final manuscript.

Funding: The work was supported by the financial supports from National Natural Science Foundation of China (No. 31571909).

Conflicts of Interest: The authors declare no conflicts of interest. 


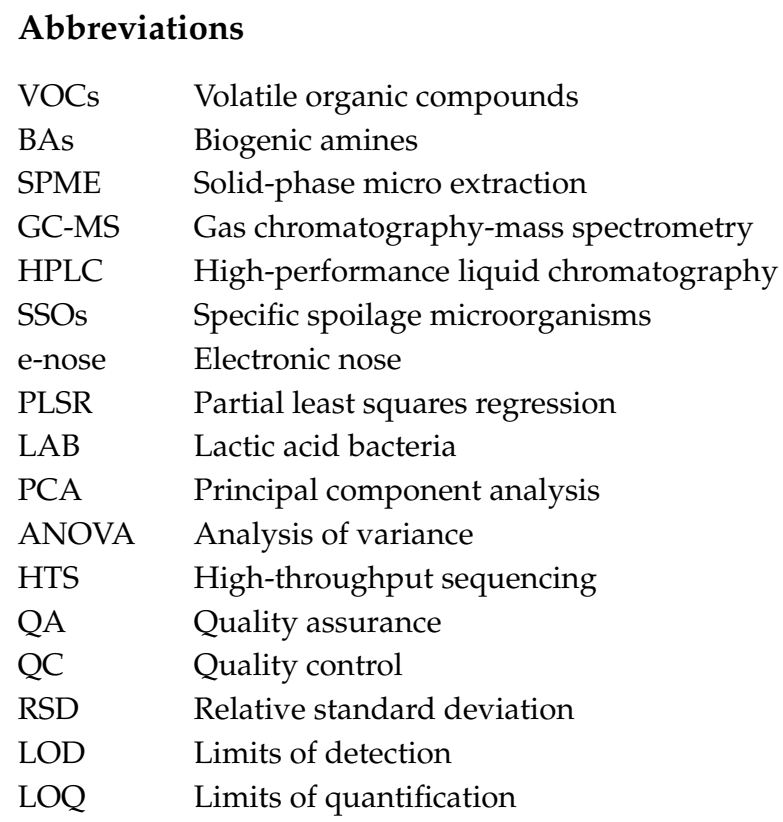

\section{References}

1. Liu, X.; Huang, Z.; Jia, S.; Zhang, J.; Li, K.; Luo, Y. The roles of bacteria in the biochemical changes of chill-stored bighead carp (Aristichthys nobilis): Proteins degradation, biogenic amines accumulation, volatiles production, and nucleotides catabolism. Food Chem. 2018, 255, 174-181. [CrossRef]

2. Casaburi, A.; Piombino, P.; Nychas, G.J.; Villani, F.; Ercolini, D. Bacterial populations and the volatilome associated to meat spoilage. Food Microbiol. 2015, 45, 83-102. [CrossRef] [PubMed]

3. Gram, L.; Huss, H.H. Microbiological spoilage of fish and fish products. Int. J. Food Microbiol. 1996, 33, $121-137$. [CrossRef]

4. Montel, M.C.; Masson, F.; Talon, R. Bacterial role in flavour development. Meat Sci. 1998, 49, 111-123. [CrossRef]

5. Huis in't Veld, J.H. Microbial and biochemical spoilage of foods: An overview. Int. J. Food Microbiol. 1996, 33, 1-18. [CrossRef]

6. Leroy, F.; Vasilopoulos, C.; Van Hemelryck, S.; Falony, G.; De Vuyst, L. Volatile analysis of spoiled, artisan-type, modified-atmosphere-packaged cooked ham stored under different temperatures. Food Microbiol. 2009, 26, 94-102. [CrossRef] [PubMed]

7. Samelis, J.; Kakouri, A.; Rementzis, J. Selective effect of the product type and the packaging conditions on the species of lactic acid bacteria dominating the spoilage microbial association of cooked meats at 4 degrees C. Food Microbiol. 2000, 17, 329-340. [CrossRef]

8. Jørgensen, L.V.; Huss, H.H.; Dalgaard, P. Significance of volatile compounds produced by spoilage bacteria in vacuum-packed cold-smoked salmon (Salmo salar) analyzed by GC-MS and multivariate regression. J. Agric. Food Chem. 2001, 49, 2376-2381. [CrossRef]

9. Carrapiso, A.I.; Jurado, Á.; Timón, M.L.; García, C. Odor-active compounds of iberian hams with different aroma characteristics. J. Agric. Food Chem. 2002, 50, 6453-6458. [CrossRef]

10. Ercolini, D.; Ferrocino, I.; Nasi, A.; Ndagijimana, M.; Vernocchi, P.; La Storia, A.; Laghi, L.; Mauriello, G.; Guerzoni, M.E.; Villani, F. Monitoring of microbial metabolites and bacterial diversity in beef stored under different packaging conditions. Appl. Environ. Microbiol. 2011, 77, 7372-7381. [CrossRef]

11. Gram, L.; Ravn, L.; Rasch, M.; Bruhn, J.B.; Christensen, A.B.; Givskov, M. Food spoilage-Interactions between food spoilage bacteria. Int. J. Food Microbiol. 2002, 78, 79-97. [CrossRef]

12. Sun, W.; Zhao, Q.; Zhao, H.; Zhao, M.; Yang, B. Volatile compounds of cantonese sausage released at different stages of processing and storage. Food Chem. 2010, 121, 319-325. [CrossRef]

13. Hernandezjover, T.; Izquierdopulido, M.; Veciananogues, M.T.; Marinefont, A.; Vidalcarou, M.C. Biogenic amine and polyamine contents in meat and meat products. J. Agric. Food Chem. 1997, 45, 2098-2102. [CrossRef] 
14. Loizzo, M.R.; Spizzirri, U.G.; Bonesi, M.; Tundis, R.; Picci, N.; Restuccia, D. Influence of packaging conditions on biogenic amines and fatty acids evolution during 15 months storage of a typical spreadable salami ('nduja). Food Chem. 2016, 213, 115-122. [CrossRef] [PubMed]

15. De Mey, E.; De Klerck, K.; De Maere, H.; Dewulf, L.; Derdelinckx, G.; Peeters, M.-C.; Fraeye, I.; Vander Heyden, Y.; Paelinck, H. The occurrence of n-nitrosamines, residual nitrite and biogenic amines in commercial dry fermented sausages and evaluation of their occasional relation. Meat Sci. 2014, 96, 821-828. [CrossRef] [PubMed]

16. Chenoll, E.; Macian, M.C.; Elizaquivel, P.; Aznar, R. Lactic acid bacteria associated with vacuum-packed cooked meat product spoilage: Population analysis by rDNA-based methods. J. Appl. Microbiol. 2007, 102, 498-508. [CrossRef] [PubMed]

17. Mataragas, M.; Skandamis, P.; Nychas, G.J.; Drosinos, E.H. Modeling and predicting spoilage of cooked, cured meat products by multivariate analysis. Meat Sci. 2007, 77, 348-356. [CrossRef] [PubMed]

18. Mansur, A.R.; Song, E.J.; Cho, Y.S.; Nam, Y.D.; Choi, Y.S.; Kim, D.O.; Seo, D.H.; Nam, T.G. Comparative evaluation of spoilage-related bacterial diversity and metabolite profiles in chilled beef stored under air and vacuum packaging. Food Microbiol. 2019, 77, 166-172. [CrossRef] [PubMed]

19. Buňková, L.; Buňka, F.; Klčovská, P.; Mrkvička, V.; Doležalová, M.; Kráčmar, S. Formation of biogenic amines by Gram-negative bacteria isolated from poultry skin. Food Chem. 2010, 121, 203-206. [CrossRef]

20. Olafsdottir, G.; Jonsdottir, R.; Lauzon, H.L.; Luten, J.; Kristbergsson, K. Characterization of volatile compounds in chilled cod (Gadus morhua) fillets by gas chromatography and detection of quality indicators by an electronic nose. J. Agric. Food Chem. 2005, 53, 10140-10147. [CrossRef]

21. Jaffrès, E.; Lalanne, V.; Macé, S.; Cornet, J.; Cardinal, M.; Sérot, T.; Dousset, X.; Joffraud, J.-J. Sensory characteristics of spoilage and volatile compounds associated with bacteria isolated from cooked and peeled tropical shrimps using SPME-GC-MS analysis. Int. J. Food Microbiol. 2011, 147, 195-202. [CrossRef] [PubMed]

22. Liu, F.; Song, S.; Zhang, X.; Tan, C.; Karangwa, E. Effect of sterilization methods on ginger flavor beverage assessed by partial least squares regression of descriptive sensory analysis and gas chromatography-mass spectrometry. Eur. Food Res. Technol. 2013, 238, 247-257. [CrossRef]

23. Blixt, Y.; Borch, E. Using an electronic nose for determining the spoilage of vacuum-packaged beef. Int. J. Food Microbiol. 1999, 46, 123-134. [CrossRef]

24. Hansen, T.; Petersen, M.A.; Byrne, D.V. Sensory based quality control utilising an electronic nose and GC-MS analyses to predict end-product quality from raw materials. Meat Sci. 2005, 69, 621-634. [CrossRef] [PubMed]

25. Boothe, D.D.; Arnold, J.W. Electronic nose analysis of volatile compounds from poultry meat samples, fresh and after refrigerated storage. J. Sci. Food Agric. 2002, 82, 315-322. [CrossRef]

26. Pennazza, G.; Fanali, C.; Santonico, M.; Dugo, L.; Cucchiarini, L.; Dachà, M.; D'Amico, A.; Costa, R.; Dugo, P.; Mondello, L. Electronic nose and GC-MS analysis of volatile compounds in tuber magnatum pico: Evaluation of different storage conditions. Food Chem. 2013, 136, 668-674. [CrossRef] [PubMed]

27. Cosio, M.S.; Ballabio, D.; Benedetti, S.; Gigliotti, C. Evaluation of different storage conditions of extra virgin olive oils with an innovative recognition tool built by means of electronic nose and electronic tongue. Food Chem. 2007, 101, 485-491. [CrossRef]

28. Labreche, S.; Bazzo, S.; Cade, S.; Chanie, E. Shelf life determination by electronic nose: Application to milk. Sens. Actuators B Chem. 2005, 106, 199-206. [CrossRef]

29. Siegmund, B.; Pfannhauser, W. Changes of the volatile fraction of cooked chicken meat during chill storing: Results obtained by the electronic nose in comparison to GC-MS and GC olfactometry. Z. Lebensm. Forsch. A 1999, 208, 336-341. [CrossRef]

30. Arnold, J.W.; Senter, S.D. Use of digital aroma technology and SPME GC-MS to compare volatile compounds produced by bacteria isolated from processed poultry. J. Sci. Food Agric. 1998, 78, 343-348. [CrossRef]

31. Lin, S.; Zhang, X. A rapid and novel method for predicting nicotine alkaloids in tobacco through electronic nose and partial least-squares regression analysis. Anal. Methods 2016, 8, 1609-1617. [CrossRef]

32. Xiao, Z.; Wu, M.; Niu, Y.; Chen, F.; Zhang, X.; Zhu, J.; Song, S.; Zhu, G. Contribution of chicken base addition to aroma characteristics of maillard reaction products based on gas chromatography-mass spectrometry, electronic nose, and statistical analysis. Food Sci. Biotechnol. 2015, 24, 411-419. [CrossRef]

33. Soladoye, P.O.; Shand, P.J.; Aalhus, J.L.; Gariépy, C.; Juárez, M. Review: Pork belly quality, bacon properties and recent consumer trends. Can. J. Anim. Sci. 2015, 95, 325-340. [CrossRef] 
34. Ghasemi-Varnamkhasti, M.; Mohtasebi, S.S.; Siadat, M.; Balasubramanian, S. Meat quality assessment by electronic nose (machine olfaction technology). Sensors 2009, 9, 6058-6083. [CrossRef] [PubMed]

35. Peris, M.; Escuder-Gilabert, L. A 21st century technique for food control: Electronic noses. Anal. Chim. Acta 2009, 638, 1-15. [CrossRef] [PubMed]

36. Alpha MOS Electronic Noses. Available online: https://www.norlab.com/infocenter/207 (accessed on 9 September 2018).

37. Li, X.; Li, C.; Ye, H.; Wang, Z.; Wu, X.; Han, Y.; Xu, B. Changes in the microbial communities in vacuum-packaged smoked bacon during storage. Food Microbiol. 2019, 77, 26-37. [CrossRef] [PubMed]

38. Hinrichsen, L.L.; Pedersen, S.B. Relationship among flavor, volatile compounds, chemical changes, and microflora in Italian-type dry-cured ham during processing. J. Agric. Food Chem. 1995, 43, 2932-2940. [CrossRef]

39. Olivares, A.; Navarro, J.L.; Flores, M. Establishment of the contribution of volatile compounds to the aroma of fermented sausages at different stages of processing and storage. Food Chem. 2009, 115, 1464-1472. [CrossRef]

40. Montanari, C.; Bargossi, E.; Gardini, A.; Lanciotti, R.; Magnani, R.; Gardini, F.; Tabanelli, G. Correlation between volatile profiles of italian fermented sausages and their size and starter culture. Food Chem. 2016, 192, 736-744. [CrossRef]

41. Ansorena, D.; Gimeno, O.; Astiasaran, I.J. Analysis of volatile compounds by GC-MS of a dry fermented sausage: Chorizo de Pamplona. Food Res. Int. 2001, 34, 67-75. [CrossRef]

42. Gamerschlag, R.; Möller, K.; Verbeeten, F. Determinants of voluntary CSR disclosure: Empirical evidence from Germany. Rev. Manag. Sci. 2010, 5, 233-262. [CrossRef]

43. Mottram, D.S. Flavour formation in meat and meat products: A review. Food Chem. 1998, 62, 415-424. [CrossRef]

44. Rivas-Cañedo, A.; Juez-Ojeda, C.; Nuñez, M.; Fernández-García, E. Effects of high-pressure processing on the volatile compounds of sliced cooked pork shoulder during refrigerated storage. Food Chem. 2011, 124, 749-758. [CrossRef]

45. Yu, A.-N.; Sun, B.-G.; Tian, D.-T.; Qu, W.-Y. Analysis of volatile compounds in traditional smoke-cured bacon (CSCB) with different fiber coatings using spme. Food Chem. 2008, 110, 233-238. [CrossRef] [PubMed]

46. Sabio, E.; Vidal-Aragón, M.; Bernalte, M.; Gata, J. Volatile compounds present in six types of dry-cured ham from south European countries. Food Chem. 1998, 61, 493-503. [CrossRef]

47. Wang, S.; He, Y.; Wang, Y.; Tao, N.; Wu, X.; Wang, X.; Qiu, W.; Ma, M. Comparison of flavour qualities of three sourced eriocheir sinensis. Food Chem. 2016, 200, 24-31. [CrossRef] [PubMed]

48. Shahidi, F.; Rubin, L.J.; D'Souza, L.A. Meat flavor volatiles: A review of the composition, techniques of analysis, and sensory evaluation. Crit. Rev. Food Sci. Nutr. 1986, 24, 141-243. [CrossRef] [PubMed]

49. Ordonez, J.A.; Hierro, E.M.; Bruna, J.M.; de la Hoz, L. Changes in the components of dry-fermented sausages during ripening. Crit. Rev. Food Sci. Nutr. 1999, 39, 329-367. [CrossRef] [PubMed]

50. Kjällstrand, J.; Ramnäs, O.; Petersson, G. Methoxyphenols from burning of Scandinavian forest plant materials. Chemosphere 2000, 41, 735-741. [CrossRef]

51. Jerković, I.; Kovačević, D.; Šubarić, D.; Marijanović, Z.; Mastanjević, K.; Suman, K. Authentication study of volatile flavour compounds composition in Slavonian traditional dry fermented salami "kulen". Food Chem. 2010, 119, 813-822. [CrossRef]

52. Chen, Q.; Kong, B.; Han, Q.; Xia, X.; Xu, L. The role of bacterial fermentation in lipolysis and lipid oxidation in Harbin dry sausages and its flavour development. LWT-Food Sci. Technol. 2017, 77, 389-396. [CrossRef]

53. Toldra', F. Proteolysis and lipolysis in flavour development of dry-cured meat products. Meat Sci. 1998, 49, $101-110$. [CrossRef]

54. Zotta, T.; Ricciardi, A.; Ianniello, R.G.; Storti, L.V.; Glibota, N.A.; Parente, E. Aerobic and respirative growth of heterofermentative lactic acid bacteria: A screening study. Food Microbiol. 2018, 76, 117-127. [CrossRef] [PubMed]

55. Jones, R.J. Observations on the succession dynamics of lactic acid bacteria populations in chill-stored vacuum-packaged beef. Int. J. Food Microbiol. 2004, 90, 273-282. [CrossRef]

56. Martín, A.; Benito, M.J.; Aranda, E.; Ruiz-Moyano, S.; Córdoba, J.J.; Córdoba, M.G. Characterization by volatile compounds of microbial deep spoilage in Iberian dry-cured ham. J. Food Sci. 2010, 75, 360-365. [CrossRef] [PubMed]

57. Joffraud, J.J.; Leroi, F.; Roy, C.; Berdagué, J.L. Characterization of volatile compounds produced by bacteria isolated from cold-smoked salmon flora. Inter. J. Food Microbiol. 2001, 66, 175-184. [CrossRef] 
58. Cui, S.; Wang, J.; Yang, L.; Wu, J.; Wang, X. Qualitative and quantitative analysis on aroma characteristics of ginseng at different ages using E-nose and GC-MS combined with chemometrics. J. Pharm. Biomed. Anal. 2015, 102, 64-77. [CrossRef] [PubMed]

59. Cmg, S.; Mba, G. Bioactive amines in chicken breast and thigh after slaughter and during storage at $4 \pm 1^{\circ} \mathrm{C}$ and in chicken-based meat products. Food Chem. 2002, 78, 241-248.

60. Kalač, P. Biologically active polyamines in beef, pork and meat products: A review. Meat Sci. 2006, 73, 1-11. [CrossRef]

61. Dainty, R.H.; Edwards, R.A.; Hibbard, C.M.; Ramantanis, S.V. Bacterial sources of putrescine and cadaverine in chill stored vacuum-packaged beef. J. Appl. Bacteriol. 2010, 61, 117-123. [CrossRef]

62. Lonvaud-Funel, A. Biogenic amines in wines: Role of lactic acid bacteria. FEMS Microbiol. Lett. 2001, 199, 9-13. [CrossRef] [PubMed]

63. Bover-Cid, S.; Izquierdo-Pulido, M.; Carmen Vidal-Carou, M. Changes in biogenic amine and polyamine contents in slightly fermented sausages manufactured with and without sugar. Meat Sci. 2001, 57, 215-221. [CrossRef]

64. Niu, Y.; Zhang, X.; Xiao, Z.; Song, S.; Eric, K.; Jia, C.; Yu, H.; Zhu, J. Characterization of odor-active compounds of various cherry wines by gas chromatography-mass spectrometry, gas chromatography-olfactometry and their correlation with sensory attributes. J. Chromatogr. B 2011, 879, 2287-2293. [CrossRef] [PubMed]

65. Song, S.; Zhang, X.; Hayat, K.; Huang, M.; Liu, P.; Karangwa, E.; Gu, F.; Jia, C.; Xia, S.; Xiao, Z.; Niu, Y. Contribution of beef base to aroma characteristics of beeflike process flavour assessed by descriptive sensory analysis and gas chromatography olfactometry and partial least squares regression. J. Chromatogr. Coruña 2010, 1217, 7788-7799. [CrossRef]

66. Tian, H.; Zhan, P.; Li, W.; Zhang, X.; He, X.; Ma, Y.; Guo, Z.; Zhang, D. Contribution to the aroma characteristics of mutton process flavor from oxidized suet evaluated by descriptive sensory analysis, gas chromatography, and electronic nose through partial least squares regression. Eur. J. Lipid Sci. Technol. 2014, 116, 1522-1533. [CrossRef]

67. Dainty, R.H.; Mackey, B.M. The relationship between the phenotypic properties of bacteria from chill-stored meat and spoilage processes. J. Appl. Bacteriol. 1992, 73, 103-114. [CrossRef]

68. Ercolini, D.; Russo, F.; Torrieri, E.; Masi, P.; Villani, F. Changes in the spoilage-related microbiota of beef during refrigerated storage under different packaging conditions. Appl. Environ. Microbiol. 2006, 72, 4663-4671. [CrossRef]

69. Tsigarida, E.; Nychas, G.-J.E. Ecophysiological attributes of a lactobacillus sp. and a Pseudomonas sp. on sterile beef fillets in relation to storage temperature and film permeability. J. Appl. Microbiol. 2001, 90, 696-705. [CrossRef]

70. Doulgeraki, A.I.; Paramithiotis, S.; Kagkli, D.M.; Nychas, G.-J.E. Lactic acid bacteria population dynamics during minced beef storage under aerobic or modified atmosphere packaging conditions. Food Microbiol. 2010, 27, 1028-1034. [CrossRef]

71. Alpha MOS. STREE Electrochemical Sensor Technology. Available online: http://www.alphamos-cn.com/ (accessed on 9 September 2018).

72. Ngapo, T.M.; Vachon, L. Biogenic amine concentrations and evolution in "chilled" Canadian pork for the Japanese market. Food Chem. 2017, 233, 500-506. [CrossRef]

73. Zhu, J.; Chen, F.; Wang, L.; Niu, Y.; Shu, C.; Chen, H.; Xiao, Z. Comparison of aroma-active compounds and sensory characteristics of durian (Durio zibethinusl.) wines using strains ofsaccharomyces cerevisiaewith odor activity values and partial least-squares regression. J. Agric. Food Chem. 2015, 63, 1939-1947. [CrossRef] [PubMed]

74. Kim, M.-K.; Mah, J.-H.; Hwang, H.-J. Biogenic amine formation and bacterial contribution in fish, squid and shellfish. Food Chem. 2009, 116, 87-95. [CrossRef]

Sample Availability: Samples of the compounds are not available from the authors. 